\title{
Understanding and Leveraging the Impact of Response Latency on User Behaviour in Web Search
}

\author{
XIAO BAI, Yahoo Research \\ IOANNIS ARAPAKIS*, Telefonica Research \\ B. BARLA CAMBAZOGLU*, NTENT \\ ANA FREIRE, Universitat Pompeu Fabra
}

\begin{abstract}
The interplay between the response latency of web search systems and users' search experience has only recently started to attract research attention, despite the important implications of response latency on monetisation of such systems. In this work, we carry out two complementary studies to investigate the impact of response latency on users' searching behaviour in web search engines. We first conduct a controlled user study to investigate the sensitivity of users to increasing delays in response latency. This study shows that the users of a fast search system are more sensitive to delays than the users of a slow search system. Moreover, the study finds that users are more likely to notice the response latency delays beyond a certain latency threshold, their search experience potentially being affected. We then analyse a large number of search queries obtained from Yahoo Web Search to investigate the impact of response latency on users' click behaviour. This analysis demonstrates the significant change in click behaviour as the response latency increases. We also find that certain user, context, and query attributes play a role in the way increasing response latency affects the click behaviour. To demonstrate a possible use case for our findings, we devise a machine learning framework that leverages the latency impact, together with other features, to predict whether a user will issue any clicks on web search results. As a further extension of this use case, we investigate whether this machine learning framework can be exploited to help search engines reduce their energy consumption during query processing.
\end{abstract}

CCS Concepts: • Information systems $\rightarrow$ Web search engines; Information retrieval; Query log analysis; $\bullet$ Human-centered computing $\rightarrow$ User studies; Laboratory experiments;

Additional Key Words and Phrases: Web search engine, response latency, user behaviour, search experience, user engagement, click prediction, energy consumption, green information retrieval

ACM Reference format:

Xiao Bai, Ioannis Arapakis, B. Barla Cambazoglu, and Ana Freire. 2017. Understanding and Leveraging the Impact of Response Latency on User Behaviour in Web Search. ACM Transactions on Information Systems 9, 4, Article 39 (March 2017), 43 pages.

https://doi.org/0000001.0000001

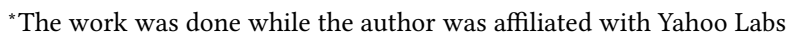

This work is an extension of an earlier work published by the authors [1]

Author's addresses: X. Bai, Yahoo Research, 701 1st Avenue, Sunnyvale, CA, 94089, USA; I. Arapakis, Telefonica Research, Plaça d'Ernest Lluch i Martin 5, Barcelona, 08019, Spain; B. Barla Cambazoglu, NTENT, Barcelona, Spain; A. Freire, Universitat Pompeu Fabra, Carrer de Roc Boronat 138, Barcelona, 08018, Spain.

Permission to make digital or hard copies of all or part of this work for personal or classroom use is granted without fee provided that copies are not made or distributed for profit or commercial advantage and that copies bear this notice and the full citation on the first page. Copyrights for components of this work owned by others than the author(s) must be honored. Abstracting with credit is permitted. To copy otherwise, or republish, to post on servers or to redistribute to lists, requires prior specific permission and/or a fee. Request permissions from permissions@acm.org.

(C) 2017 Copyright held by the owner/author(s). Publication rights licensed to Association for Computing Machinery.

1046-8188/2017/3-ART39 \$15.00

https://doi.org/0000001.0000001

ACM Transactions on Information Systems, Vol. 9, No. 4, Article 39. Publication date: March 2017. 


\section{INTRODUCTION}

Serving search results too slow or too fast both may result in certain financial consequences for a search engine. On the user side, the new generation of web users are impatient and have limited time. They expect sub-second response times from a search engine upon submission of their queries. High response latency is known to distract users and cause them to issue fewer queries than usual, decreasing users' engagement with the search engine in the long term [42]. This, in turn, can make a negative impact on the advertising revenue of the search engine. On the system side, commercial web search companies are known to make major investments in hardware infrastructures to cope with the growth of the Web as well as the growth of their user bases and query volumes, essentially trying to maintain their query response times at reasonable levels [10]. These investments incur a financial burden on search engine companies and may even result in financial losses if the reduction attained in query response times due to these investments does not have any positive impact on the search experience of users.

In this paper, we investigate both the user and system aspects of the response latency issue in web search engines. Our first line of research focuses on understanding the potential impact of response latency on users' search behaviour. In particular, our work aims to answer questions of the following kind.

- RQ1. What are the main cost components in the response latency of a web search engine (Section 3.2) and how is its distribution (Section 3.3)?

- RQ2. How sensitive are the users to increasing delays in search engine response (Section 4.1) and how does latency affect users' search experience (Section 4.3)?

- RQ3. What is the effect of increasing response latency on the click behaviour of users (Section 5.2)?

- RQ4. How do different demographic groups respond to increasing response latency (Section 5.3)?

- RQ5. In what kind of context the users are less tolerant to slow response (Sections 5.4 and 5.5)?

Our second line of research deals with the implications on the system side. Here, we try to answer questions of the following kind.

- RQ6. Can we exploit any prior knowledge about the user-perceived response latency to infer users' click behaviour (Section 6)?

- RQ7. If this turns out to be feasible, can we devise a method to use the results of this inference to achieve energy savings in web search engines (Section 7)?

The contributions of our work are summarised below. We note that the last three contributions are new and do not appear in [1], which the current paper extends.

- First, we describe the dominant factors in web search response latency and demonstrate the relative importance of each factor using real-life data traces.

- Second, we conduct a small-scale, controlled user study, which reveals the differences in the way users perceive the latency.

- Third, we conduct a large-scale query log analysis using search queries obtained from Yahoo Web Search, providing certain insights about the impact of increasing response latency on the click behaviour of users in general.

- Fourth, we identify important user, time, and query attributes and study their impact on the click behaviour of users in face of different or similar search response latencies through large-scale query log analysis.

- Fifth, we propose a machine-learned click prediction framework that exploits individual latency components as well as certain user, time, and query attributes identified in our query 
$\log$ analysis as features. We demonstrate that we can anticipate with reasonable accuracy whether a user will issue any click on web search results or not using these features.

- Sixth, we present a technique that uses the proposed click prediction framework to reduce the energy consumption of web search engines through early termination of the processing of queries that are predicted to not receive any click in the case of high response latency.

The selected findings of our work are the following.

- Query processing and result page rendering times are the two main components in the response latency of a web search engine. Network latency becomes more pronounced as the end-to-end latency increases.

- The users of a fast search system are more likely to notice the delays in latency than the users of a slow search system.

- As long as the delay added to a response remains under $500 \mathrm{~ms}$, users cannot distinguish between a delayed response and a regular response with no added delay. When the introduced delay is larger than $1000 \mathrm{~ms}$, users are very likely to notice the presence of a delay.

- Given two content-wise identical search result pages, users are more likely to perform clicks on the result page that is served with lower latency.

- The degree to which response latency affects click behaviour varies according to user attributes. For example, females and elders can tolerate high latency better.

- It is possible to accurately predict whether a user will issue any click on web search results or not, relying on simple features extracted from the response latency, user, query, and context.

- Terminating query processing, based on the predicted click likelihood at increasing latency intervals, can lead to reduction in energy consumption of search engines.

The rest of the paper is organised as follows. We survey the related work in Section 2. In Section 3, we provide some initial experiments aiming to characterise the response latency of a web search engine. The details and findings of our controlled user study are presented in Section 4. In Section 5, we present our large-scale query log analysis. In Section 6, we present a machine learning framework to predict whether a user will issue any click on a search engine result page by exploiting a subset of the features investigated in Section 5. In Section 7, we investigate the feasibility of using this machine learning framework to reduce the energy consumption of a web search engine. The paper is concluded in Section 8.

\section{RELATED WORK}

\subsection{Cost of Searching}

A related line of research has investigated the trade-off between the cost of searching and user effectiveness in interactive information retrieval. In [43], the querying cost was represented by the physical or mental effort spent by the users when searching for certain information in a retrieval system. In [2], the microeconomic theory was applied to interactive information retrieval, and it was shown that useful information obtained by a user during a search session is functionally related to the effort spent by issuing queries and examining retrieved results. In [3], the authors conducted a user study where participants were split into three groups to use different search interfaces, each requiring a different amount of physical and mental effort for issuing queries. Although most results reported by the study were not statistically significant, the authors observed that the participants who used the search interface with high querying cost submitted fewer queries, examined more result documents per query, and spent more time on search result pages. In [35], the authors verified the validity of five different hypotheses (taken from information foraging and search economic theories) about how users' search behaviour should change when faced with 
delays. The study involved 48 participants who interacted with four different search interfaces with different types of delays (no delay, only query response delay, only document download delay, and both query response and document download delays). The study found strong support for the three of the hypotheses. In [6], the authors simulated interactive search sessions assuming a desktop PC scenario, where querying effort is low, and a smart phone scenario, which requires high querying effort. They showed that the user effort spent on searching, when coupled with a time constraint on the session duration, affected the user experience in both scenarios. In particular, they found that the smart phone scenario led to deeper result scanning while the desktop PC scenario favoured better queries.

\subsection{Metrics}

Certain effectiveness metrics, such as DCG [28] and RBP [38], incorporated the user effort implicitly by decaying the information gain with increasing rank (assuming users scan search results from top to bottom and spend a fixed amount of effort when examining each result). The time-based gain measure in [44] incorporated the user effort more explicitly by using the time spent scanning the results.

\subsection{Page Load Time}

There are a number of studies on the response time of general computer systems in the context of human-computer interaction. The reader may refer to [15] for a discussion of those studies. In the more specific context of web systems, earlier studies investigated the impact of page load time on the web browsing behaviour of users [16-18, 21, 26, 39, 41, 45]. The study in [45] (follow-up work to [18]) reported web page load time tolerable by users who are seeking information in the Web to be in the 7 to 11 seconds range. The same study showed that there is a latency threshold at which users start examining the content of web pages more thoroughly before navigating to new pages. Although the context is different, this finding is consistent with the cost-interaction hypothesis, which states that users examine search results in more depth before issuing queries when the querying effort is high [3]. Despite being outdated, [39] provides extensive references to studies on identifying the largest page load time that users can tolerate.

\subsection{Query Response Latency}

In [42], the authors exposed a commercial search engine's users to response time delays of varying magnitude and observed the impact of different levels of delay on users' long-term search behaviour. They observed that the users who were exposed to higher time delays issued fewer queries than they usually do. Interestingly, the effects were shown to be persistent in the long-term even after the response latency had returned to the original levels. Our work differs from [42] in two ways. First, our user study allows us to introduce artificial response time delays on the client side, whereas [42] relies on server-side delays. This lets us work with more realistic (user-perceived) latency values and provides better control on certain parameters. Second, in our query log analysis, we focus on the short-term click behaviour of individual users, instead of the change in aggregate query volumes, which is the main metric in [42].

The most relevant work to ours is the user study presented in [8], although it differs significantly with respect to the adopted methodology. In [8], the participants interacted with two simple interfaces serving search results at controlled latency values, and stated their preferences between a slow and a fast search interface through a questionnaire. The findings of the study regarding the impact of latency on users' preferences were mainly inconclusive. In our user study, instead of assigning participants into two fixed latency buckets, we expose each participant to multiple 
levels of latency, allowing us to investigate the way they perceive the latency better. Moreover, we experiment with much lower latency levels, which are more realistic for today's web standards (our latency values range between 0 and $2750 \mathrm{~ms}$ with an increment of $250 \mathrm{~ms}$, whereas the latency values used in [8] range between 1 and 5 seconds with an increment of 1 second).

In [5], the authors conducted a controlled user study to reveal the physiological effects of response latency on users. The reported results indicate that the latency effects are present even at small increases in response latency. They complemented their user study by a query log analysis similar to ours. The user studies conducted in our work differ from those in [5] in that our work focuses on the perceivable effects of increasing response latency, while the latter work investigatesunconscious latency effects that may still effect user behaviour.

Last, the authors in [46] performed a query log analysis on the impact of increasing response latency on user engagement. Their analysis indicated that, with increasing response latency, the likelihood that the user will click on the search results decreased. Moreover, the authors observed an increase in the time the users issue the first click on the search result page as the response latency increases. Both findings point out the negative consequences of slow response on user engagement.

\subsection{Latency Prediction}

In [25], techniques are proposed to improve the response latency of a web search engine. The study focused on the reduction of tail latency by leveraging features collected at run time (dynamic prediction), enabling estimation of query execution time with higher accuracy. Using both multicore and heterogeneous processors, authors showed how this dynamic prediction can improve the performance of a search engine. In our scenario, we cannot exploit such run-time features since our experiments are based on simulations. Earlier works on latency prediction in the context of web search engines include [34], [29], and [31].

\subsection{Click Prediction}

Click prediction has a number of applications. One notable example is the domain of online advertising, which in the past years has attracted the attention of major companies such as Google [37], Facebook [24], and Yahoo [13]. In the context of web search, click information has been widely used in machine-learned ranking strategies to improve the search result quality and promote better user experience [27].

\subsection{Energy Consumption of Search Data Centres}

While several efforts have been made so far to reduce the power consumption of general-purpose data centres, few of them are actually focused on proposing efficiency improvements and designing more sustainable web search engines [14]. Previous research has addressed power consumption with respect to different web search architectures, such as geographically distributed data centres [30], replicated search engines [19,20], and single servers [11]. Some of the above approaches leverage the fluctuation in query traffic along the day; others combine workload with electricity rates to take the most suitable decision. However, to the best of our knowledge, our work is the first to use click prediction as a way to reduce the energy consumption of web search engines.

\subsection{Extensions to the Previous Version}

Our paper extends an earlier work published as a conference paper [1]. The extensions over the previous paper are three-fold. First, we analyse the interplay between search response latency and different attributes, such as gender, age, level of search activity, time of the day, query length, query 


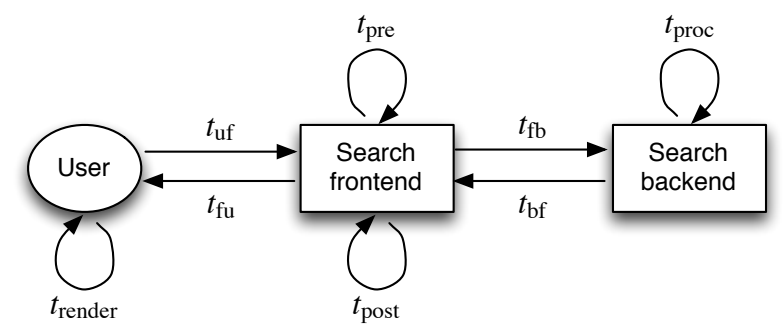

Fig. 1. The components of response latency in web search engines.

frequency, and type of information need. This fine-grain analysis complements the coarse-grain analysis in the previous paper. More importantly, this analysis shows how such attributes may impact user click behaviour in face of different search response latencies and leads to our second contribution in this work. Specifically, we devise a machine learning framework leveraging certain latency features, together with other basic attributes on the user, query and time, obtained from our fine-grain analysis, to predict whether the user will issue any click on the retrieved web search results. We then demonstrate the predictive power of this model through carefully conducted experiments. As the third contribution, we discuss the potential implications of our click prediction framework in achieving energy savings in web search engines.

\section{PRELIMINARIES}

\subsection{Retrieving Search Results}

In a typical web search scenario, a user submits a query to a search engine by typing one or more keywords into a search box. The query is then transferred over the network, from the user's device to a frontend system in the web search engine. If the results of the query are already cached in the frontend system, they can be immediately served. Otherwise, the query is transformed into an internal representation after some preprocessing (e.g., query expansion, spell correction) and communicated to one or more backend query processing systems. Each backend system identifies the best-matching search results for the query by processing an inverted index (potentially coupling the process with machine-learned ranking). The results returned by different backends are aggregated into a final search result page, which is cached in the frontend system and communicated back to the user over the network. Finally, the search results received by the user's device are rendered using a browser. The basic search process and individual latency components are illustrated in Fig. 1.

\subsection{Constituents of User-Perceived Response Latency}

In the aforementioned process, user-perceived response latency is defined as the time difference between the rendering of retrieved search results in the user's browser and the submission of the query. This end-to-end latency involves three main components: network latency, search engine latency, and browser latency. The network latency is composed of the round-trip time between the user and the web search engine frontend $\left(t_{\mathrm{uf}}+t_{\mathrm{fu}}\right)$. This latency is known to correlate well with the physical distance between the user and the search engine and, to some extent, with the available network bandwidth. The search engine latency corresponds to the time difference between the arrival of the query to the search engine and the start of results' transfer to the user 


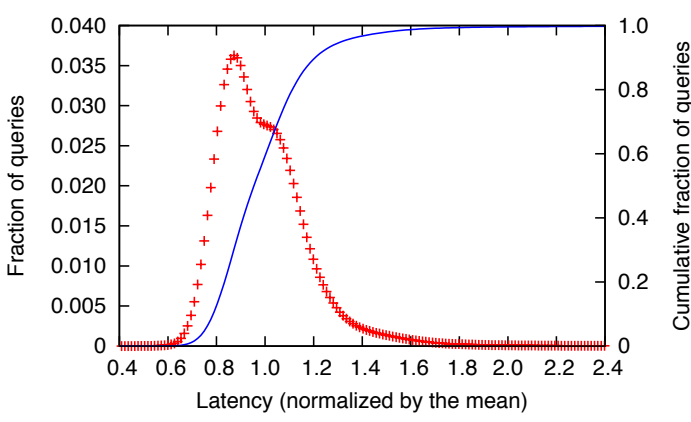

(a) Distribution of latency.

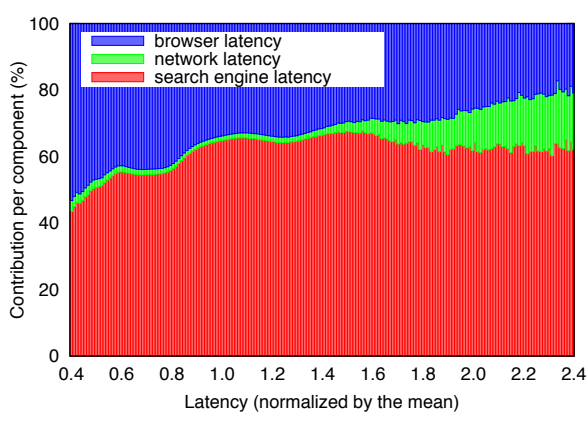

(b) Dissection of latency.

Fig. 2. Characteristics of the response latency.

$\left(t_{\mathrm{pre}}+t_{\mathrm{fb}}+t_{\mathrm{proc}}+t_{\mathrm{bf}}+t_{\mathrm{post}}\right)$. Finally, the browser latency corresponds to the time difference between the reception and rendering of search results in the user's browser $\left(t_{\text {render }}\right)$.

\subsection{Characterising Response Latency}

Fig. 2(a) shows the distribution of response latency values observed in Yahoo Web Search (please refer to Section 5.1 for the details of the query log used). ${ }^{1}$ According to the solid curve in the figure, about $60 \%$ of queries are answered under the mean latency and about $99 \%$ of queries have a response latency less than $1.8 \mu$. The latency distribution (dotted curve) is observed to have a slight distortion after the peak. This behaviour is because the distribution actually involves two sub-distributions with peaks around $0.85 \mu$ and $1.05 \mu$. The former sub-distribution is due to queries served by the result cache while the latter is due to queries processed in the main search backend.

Fig. 2(b) shows the contribution of different latency components to the user-perceived response latency. We observe that the end-to-end latency is mainly determined by the search engine latency and the browser latency. While the contribution of the two latency components are similar when the responses are fast (e.g., around $0.5 \mu$ ), the search engine latency becomes the dominant factor as the response times increase. At much larger latency values (e.g., around 1.6 $\mu$ ), the network latency starts to become more noticeable.

\subsection{Possible Experimental Methodologies}

There are three possible experimental methodologies one can adopt to carry out a study in our context: bucket testing, controlled user study, and query log analysis. In the case of bucket testing, the users of the search engine may be split into buckets, each subject to a different set of test parameters (e.g., varying added delays as in [42]). Bucket testing enables a large-scale and real-life study. However, it is not easy to control certain parameters and observe the real user experience. User studies are typically much smaller in scale [8], but a wider range of parameters can be explored in a controlled manner. The downside is the difficulty of generalising the findings. Finally, query $\log$ analysis may let us make observations using the recorded search behaviour of users. This kind of an analysis can be large scale, but has little flexibility for introducing new parameters. In our work, we adopted the user study (Section 4) and query log analysis options (Section 5).

${ }^{1}$ Due to the confidential nature of the data, the reported response latency values have been normalised by the mean latency $(\mu)$. 


\section{CONTROLLED USER STUDY}

To demonstrate the impact of response latency on search behaviour we carried out two controlled experiments that examine users' interactions with two different search sites. The first experiment investigates users' sensitivity to different levels of latency, as well as their perception of response time. The second experiment demonstrates the effects of increasing response latency on the search experience and, more specifically, on user engagement and satisfaction. In addition, we looked at potential bias due to search site branding.

\subsection{User Sensitivity to Latency}

4.1.1 Experimental Design. The experiment consisted of two types of tasks and used a repeatedmeasures design with three independent variables: adjusted latency (with 8 levels in milliseconds: “0”, “250”, “500”, “750”, “1000”, "1250”, "1500", “1750”), fixed latency (with 10 levels in milliseconds: “500”, “750”, “1000”, “1250”, “1500”, “1750”, “2000”, “2250”, “2500”, “2750”), and search site speed (with two levels: "slow", "fast").

The adjusted latency was controlled through a client-side script that adjusted the search latency experienced by the participants by a desired amount of delay. The fixed latency was controlled in a similar manner, by fixing the search latency experienced by the participants to a desired amount of delay. The two types of latencies differ with respect the following aspects: (1) how the final search latency is set (by adding to the base latency a fixed amount or by adding to the base latency a variable amount to fix it to a value) and (2) their range of values. The combined sets of latency ranges of the adjusted latency and fixed latency is a set with 12 values ("0", " 250 ", " 500 ", " 750 ", "1000", “1250”, “1500”, “1750”, “2000”, “2250”, “2500”, “2750”).

The search site speed was controlled by completing the study using two different commercial search sites: one with a generally slow response rate (slow SE) and one with a generally fast response rate (fast SE). The participants were aware that they were using two different search engines. When comparing the two commercial search engines, the main difference lies in the observed response rate while some minor differences can be noticed in the "look and feel" of the user interface. Finally, although the two search sites were different, the returned search results were very similar due to the nature of the queries used (see Section 4.1.5). The dependent variables were (i) sensitivity to search latency and (ii) prediction accuracy of search latency.

The scatter plot in Fig. 3 shows the response latency values observed for the slow SE and the fast SE upon submission of identical queries. We observe the slow SE to be slower than the fast SE. For almost every query submitted, the fast SE could retrieve the results with lower latency.

4.1.2 Apparatus. In our experiment, we used a desktop computer equipped with a $24^{\prime \prime}$ LCD monitor, keyboard, and mouse. In the background, we ran a custom-made JavaScript code that controlled the adjusted latency and fixed latency. The script was deployed using the Greasemonkey ${ }^{2}$ extension in a Mozilla Firefox web browser. It captured a series of browser events (e.g., mouseover, click, and keypress) and logged the unix timestamps for every query submitted and each search result page rendered in response to a query.

4.1.3 Questionnaires. At the beginning of the study, the participants were asked to fill in an entry questionnaire, which gathered background and demographic information such as age, gender, level of education, and current work status. In addition, the entry questionnaire gathered information about the participants' previous experience with online search as well as their prior expectations for a number of commercial search engines. A set of scales was developed specifically for our study

${ }^{2}$ http://www.greasespot.net 


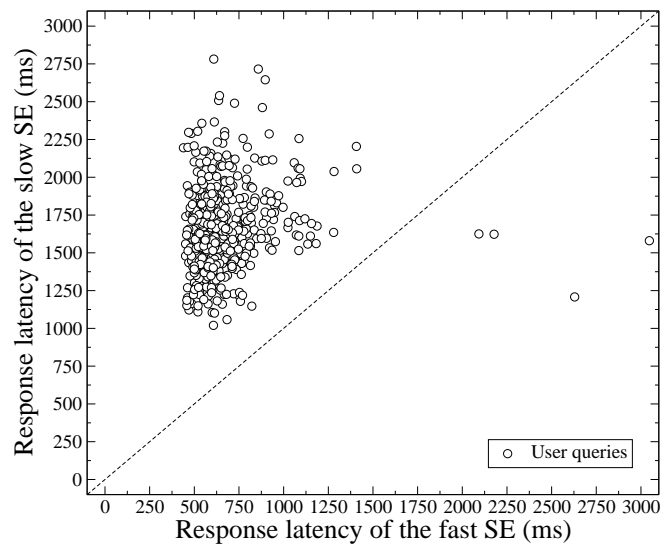

Fig. 3. Response latency values attained by the fast and slow SEs for the same query.

(e.g., easy/difficult, relaxing/stressful, and satisfying/frustrating) based on users' response to the statement "Using a search site is generally ...".

4.1.4 Participants. There were 12 participants (female $=6$, male $=6$ ) aged from 24 to 41 . The participants were of mixed nationality, came from a variety of educational backgrounds $(41.6 \% \mathrm{had}$ an MSc degree and 58.3\% had a PhD degree), and were all proficient with the English language (8\% intermediate level, $75 \%$ advanced level, $17 \%$ native speakers). They were primarily pursuing further studies while working (54.3\%) although there were a number of students $(33.3 \%)$ and fulltime employees $(16.6 \%)$. Participants reported using a search site at home or work very often $(M=6.58, S E=.79)$ and that they find online searching a very easy $(M=6.00, S E=1.53)$ and satisfying $(M=5.50, S E=1.16)$ task.

4.1.5 Procedure. The user study was carried out in a laboratory setting and followed a thinkaloud protocol. At the beginning of their session, the participants were informed about the conditions of the experiment and were asked to complete a demographics questionnaire. Then, they were asked to perform two types of tasks using both search engines (slow SE and fast SE). The goal was the same for both types of tasks: submitting a fixed number of randomly selected navigational queries, i.e., queries that seek a single website or web page of a single entity. The web domain list was created using the web analytics provided by Alexa. ${ }^{3}$

Throughout the study, participants were presented with two web browser windows: the first window displayed the search site while the second window displayed the questionnaire. For each navigational query, participants were instructed to locate the associated URL among the first ten results of the search result page and copy-paste it in the corresponding box of the questionnaire. We limited the study to navigational queries because they impose a smaller cognitive load to the searcher (compared to other types of queries), promote a convergence in the search intent across all users, and do not require native-level knowledge of the English language. Therefore, by mitigating the effort of query formulation, our participants were able to assess the latency effect better. No time limit was imposed on any of the tasks.

The first type of task asked the participants to verbally report to the experimenter their subjective impression of the search site's response latency, i.e., whether they felt that the response was "slow"

\footnotetext{
${ }^{3}$ http://www.alexa.com/topsites
} 


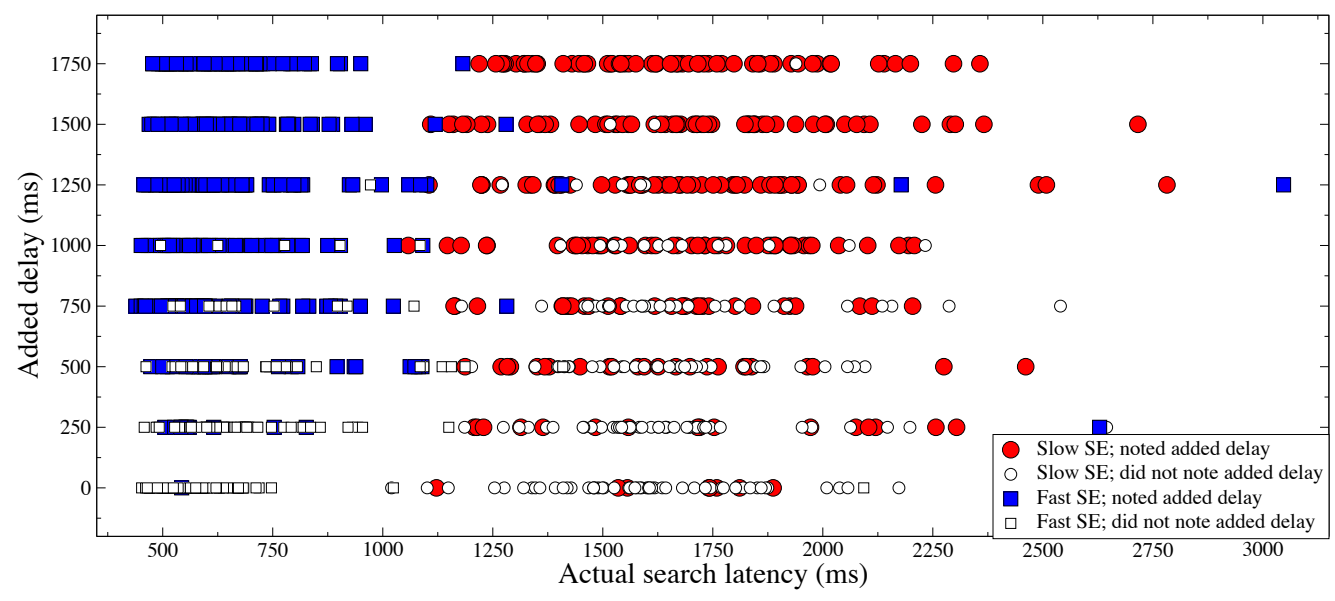

Fig. 4. Each point in the plot indicates a query for which a participant noted an added delay in the search engine response or not as the actual latency and added delay vary.

or "normal", after the search engine returned the results for each submitted query. In this type of task, we manipulated the adjusted latency variable by increasing it by a fixed amount that ranged from 0 to $1750 \mathrm{~ms}$, using a step of $250 \mathrm{~ms}$. The reason for opting for this particular variable is because we were interested in knowing whether users can perceive added latencies and can tell if the experienced search latency deviates from the "normal" search latency. Each latency value $(0 \mathrm{~ms}, 250 \mathrm{~ms}, \ldots, 1750 \mathrm{~ms})$ was introduced five times and in a random order, in combination with 40 randomly selected navigational queries (8 latency values $\times 5=40$ navigational queries). The provided navigational queries were submitted to the search site the same way they would be submitted in a realistic search scenario, i.e., through typing and clicking.

The second type of task required the participants to provide an estimation of the experienced search latency in milliseconds. More specifically, the participants were asked to report verbally to the experimenter their subjective estimation of the search latency for each submitted query. When estimating search latency, participants were instructed to consider the time difference from the query submission until the search result page was rendered. Here, we chose to manipulate the fixed latency variable by setting it to a fixed value that ranged from $500 \mathrm{~ms}$ to $2750 \mathrm{~ms}$, using a step of $250 \mathrm{~ms}$. The reason for that is because we were interested in absolute latencies and how well users would be able to predict them. Similar to the previous task, each latency value was introduced five times and in a random order, in combination with 50 navigational queries (10 latency values $\times$ $5=50$ navigational queries). To familiarise themselves with the default behaviour of the search site and establish a measure of comparison, the participants were asked to submit a set of training queries before each task.

In total, each participant performed four tasks ( 2 search engines $\times 2$ types of tasks). Finally, to control for order effects, the task assignment was randomised.

4.1.6 Results (first task). Fig. 4 shows the distribution of queries with respect to the actual search engine latency and added delay. Each data point in the figure corresponds to a query. For each query, the participant makes a prediction about whether there was an artificially introduced delay in the search engine response or not, based on the magnitude of the latency perceived by her.

Figure 5 shows the proportion of queries (i.e., likelihood) for which participants indicated they thought the system response was slow, for each search site and at each level of added delay (i.e., 


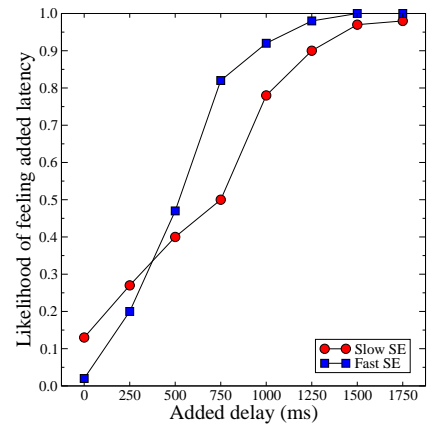

Fig. 5. Fraction of cases where participants said that they noticed added delay in the response of the slow and fast SEs, as the added delay varies.

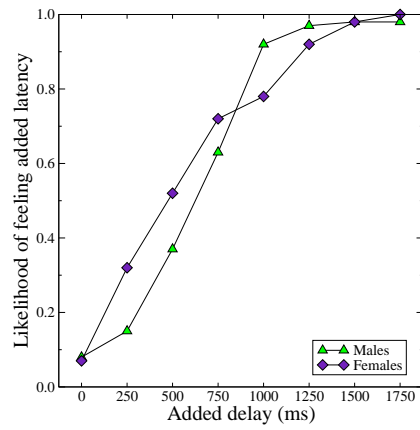

Fig. 6. Fraction of cases where male and female participants said that they noticed added delay in the response, as the added delay varies.

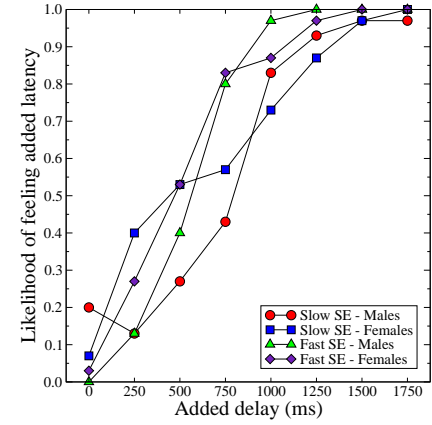

Fig. 7. Fraction of cases where male and female participants said that they noticed added delay in the response of the slow and fast SEs.

adjusted latency). Overall, more participants indicated the system was slow at higher levels of adjusted latency for both slow SE and fast SE. For adjusted latencies of 500ms and above, a higher proportion of queries were indicated as slow against fast SE than slow SE. For example, when there was no added delay, the participants of the fast SE reported correctly the absence of delay for $98 \%$ of the queries, whereas the participants of the slow SE were less likely to report the absence of delay (for $87 \%$ of the queries), potentially due to the high variation in response time of the slow SE Moreover, at $750 \mathrm{~ms}$ adjusted latency, query responses were indicated as slow for $82 \%$ of fast SE queries and only $50 \%$ of slow SE queries. This suggests that participants may be more sensitive to detecting added delay when using a system that is generally faster and has less variability in response time.

Pearson's Chi-square Test for Independence revealed a significant association $\left(\chi^{2}(7)=17.596, p<\right.$ .05 ) between the likelihood distributions and the search engine speed, i.e., whether users used the slow SE or the fast SE. This seems to represent the fact that the odds of participants noticing the added delay was higher when they were using the fast SE compared to the slow SE. For both search engines, adjusted latencies under $500 \mathrm{~ms}$ were not easily noticeable by participants (not better than random prediction) while adjusted latencies above $1000 \mathrm{~ms}$ could be noticed with very high likelihood.

Fig. 6 displays similar data, but this time accounts for the gender interaction. According to the figure, female participants reported the added delay for a higher proportion of queries than male participants. However, there is no significant difference between male and female participants for higher levels of adjusted latency. A Pearson's Chi-square Test for Independence did not reveal a significant association between gender and the likelihood of reporting the added delay. In Fig. 7 , we show similar curves for four different (search engine speed, gender) combinations.

4.1.7 Results (second task). In Figs. 8 and 9, we show the mean fixed latency values estimated by each participant over the actual experienced latency provided by the slow and fast SEs, respectively. The results reveal considerable differences in the way individuals perceive search latency. In the case of the slow SE, about half of the participants consistently overestimated the fixed latency while the other half consistently underestimated it. The prediction quality of participants have higher deviation in the case of the fast SE than in the slow SE. Interestingly, the average of all participants' predictions are very close to the original values of the fixed latency in both cases. 


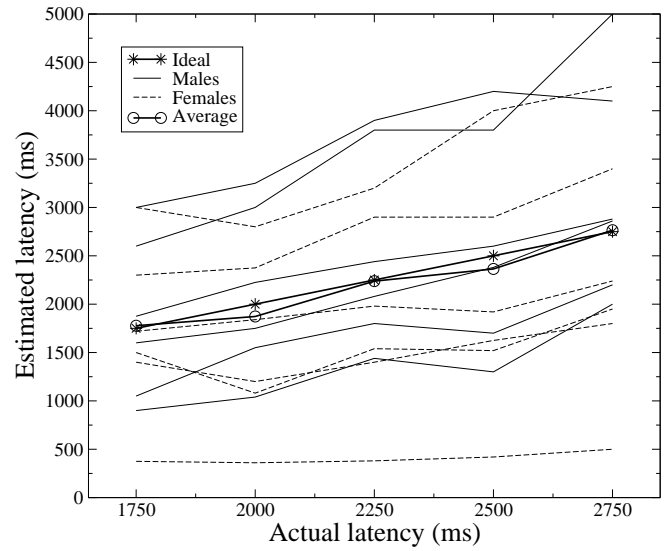

Fig. 8. Actual search engine latency (slow SE) versus the mean latency estimated by the participants.

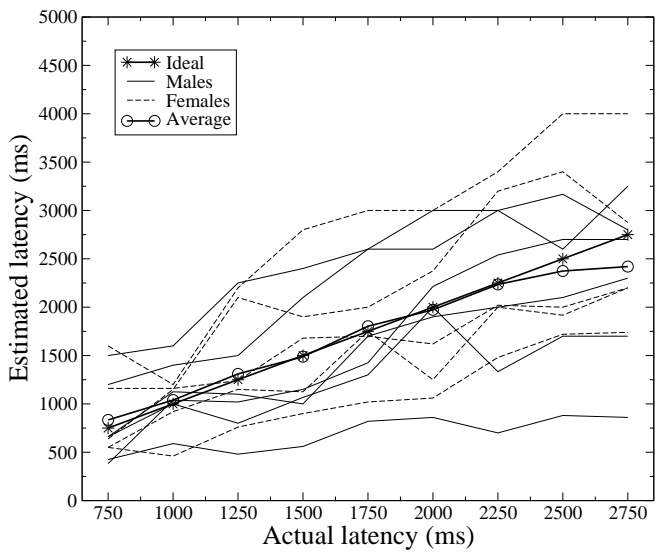

Fig. 9. Actual search engine latency (fast SE) versus the mean latency estimated by the participants.

\subsection{Summary}

One of our experiments in this section aimed at understanding whether the users can notice added delays in response latency. In response to RQ2, we found that added delays under $500 \mathrm{~ms}$ are not easily noticeable by users while those above $1000 \mathrm{~ms}$ could be noticed with very high likelihood. We also found that the users could distinguish slow response with higher likelihood when they were using a relatively fast search engine. In another experiment, we tried to see if the users can correctly predict the response latency. This experiment indicated considerable variation in the way users perceive the latency. While about a half of the users consistently overestimated the actual latency, the other half consistently underestimated.

The results reported in [8] indicate that, between a slow search engine that serves its results in more than three seconds and a fast search engine that serves its results in $250 \mathrm{~ms}$, the users are more likely to prefer the fast search engine. Our results show that such a bias appears at much lower response latency values and even smaller latency differences are noticeable by users. Most of the results reported in [8] regarding the effects of latency on user experience are inconclusive. Our findings, which focus on the short-term effects of response latency, are not comparable with those reported in [42] since the latter work evaluates the impact of response latency on user loyalty in the long term.

\subsection{Impact of Latency on Search Experience}

The objective of this study is to investigate the effects of response latency on the search experience and, in particular, on user engagement and satisfaction. Two psychometric scales were used to capture hedonic and cognitive aspects of user experience: the User Engagement Scale (UES) [40] and IBM's Computer System Usability Questionnaire (CSUQ) [32]. In addition to the psychometric scales, participants were asked to evaluate the performance and speed of the search site, as well as report the experienced frustration after each task. Our hypothesis is that, as the search latency increases, the search experience will become less engaging (i.e., low scores on all psychometric scales) and the perceived usability of the search site will be negatively impacted.

4.3.1 Experimental Design. The experiment had a two-way, mixed design. The related measures independent variable was the fixed latency (with four levels in milliseconds: "0", "750", "1250", 
"1750"). The unrelated measures independent variable was the search site speed (with two levels: "slow", "fast"). The dependent variables were (i) experienced positive and negative affect, (ii) level of focused attention, (iii) perceived system usability, and (iv) subjective beliefs about search site performance.

The fixed latency was controlled through a client-side script that adjusted the search latency experienced by the participants to a desired amount of delay. The choice of latency values was informed by the findings from the first study (see Section 4.1). The search site speed was controlled by completing the study using either a commercial search site with a generally slow response rate (slow SE) or a commercial search site with a generally fast response rate (fast SE). Despite the two search sites coming from different brands, the returned results were almost identical due to the nature of the search queries used (see Sections 4.1.1 and 4.3.5).

We note that, in this study the search site speed was designed as an unrelated measures independent variable. This is because the participants were asked to evaluate the usability of two search engines, for different latency settings. Therefore, by completing the task for one of the two search engines, they would become already familiar with the evaluation questions. This awareness of the aspects that we wanted to evaluate would have affected the spontaneity of their responses by introducing carryover effects, and thus the first task performed would adversely influence the any follow-up tasks. On the contrary, in the previous study (Section 4.1), the search site speed was designed as a related measures independent variable. The reason for that is because we wanted to test participants' sensitivity to latency and we could afford to expose them to two different search engines given task's somewhat "computational" nature of estimating latencies.

\subsubsection{Apparatus. The study had the same setup as in Section 4.1.2.}

4.3.3 Questionnaires. We used two types of questionnaires. The first questionnaire (entry) was introduced at the beginning of the study and gathered background and demographic information, as well as information about previous experience with online search. The second questionnaire (main) was administered at post-task and included the UES [40] and CSUQ [32] scales. The questions were all forced-choice type and appeared in a random sequence to mitigate potential bias due to the ordering effect. The UES [40] is multi-dimensional; its items pertain to positive and negative affect, perceived usability of the system, as well as users' felt involvement and focused attention during the task. Affect refers to the emotion mechanisms that influence our everyday interactions and can act as the primary motivation for sustaining our engagement during information processing tasks or computer-mediated activities. Focused attention refers to the feeling of energised focus and total involvement, often accompanied by loss of awareness of the outside world and distortions in the subjective perception of time.

The CSUQ [32] is a multi-dimensional user satisfaction questionnaire. Out of the four items it consists, we considered only the scores from the responses to system usefulness (SYSUSE). Taken together, the UES and CSUQ-SYSUSE probe users' perceptions of the pragmatic and hedonic qualities of their search interactions, as well as their perceptions of the search engine and of themselves using a technology, all of which are considered key facets of the user experience [23]. More specifically, the questionnaires inquired about the following aspects:

I-PANAS-SF. The international Positive and Negative Affect Schedule (PANAS) Short Form [47] was used to measure the affect before and after each task (Table 1). I-PANAS-SF is a validated test for measuring affect changes. It includes ten items measuring positive (PAS) and negative (NAS) affect. Participants were asked to respond on a 7-point Likert scale (very slightly or not at all; a little; moderately; quite a bit; extremely) their agreement to the statement: "You feel this way right now, that is, at the present moment", for each item. Although I-PANAS-SF may not be as efficient 
Table 1. I-PANAS-SF [47] (1:Very slightly or not at all; ... 7:Extremely)

\begin{tabular}{c|c}
\hline \multicolumn{2}{c}{ Question: You feel this way right now, that is, at the present moment... } \\
\hline Positive Affect Scale (PAS) & Negative Affect Scale (NAS) \\
\hline active & afraid \\
alert & ashamed \\
attentive & hostile \\
determined & nervous \\
inspired & upset \\
\hline
\end{tabular}

Table 2. FA scale [40] (1:strongly disagree; ... 7:strongly agree)

\footnotetext{
1. I forgot about my immediate surroundings while performing this search task.

2. I was so involved in my search task that I ignored everything around me.

3. I lost myself in this search experience.

4. I was so involved in my search task that I lost track of time.

5. I blocked out things around me when I was completing the search task.

6. When I was performing this search task, I lost track of this world around me.

7. The time I spent performing the search task just slipped away.

8. I was absorbed in my search task.

9. During this search task experience I let myself go.
}

Table 3. CSUQ-SYSUSE scale (1:strongly disagree; ... 7:strongly agree)

\footnotetext{
1. Overall, I am satisfied with how easy it is to use this search site.

2. I can effectively complete my work using this search site.

3. I am able to complete my work quickly using this search site.

4. I am able to efficiently complete my work using this search site.

5. I feel comfortable using this search site.

6. I believe I became productive quickly using this search site
}

and accurate for capturing temporal micro-resolutions of emotional responses, there are several examples of studies from the domain of Library \& Information Science [22, 33, 40] where PANAS has been successfully applied for measuring searchers' affect between search tasks. Considering that the duration of our search tasks is comparable to those in the aforementioned studies, we believe that our experimental approach to measuring emotion was reasonably accurate.

Focused attention. A 9-item focused attention (FA) subscale, part of the larger UES for measuring user engagement [40], was adapted to the context of the search tasks. The FA subscale has been used in past work [36] to evaluate users' perceptions of time passing and their degree of awareness about what took place outside of their interaction with the given task. Given the context of our work, FA was a more meaningful dimension, at least compared to other subscales of engagement (e.g., aesthetics, novelty) that were not relevant enough or were addressed by the other questionnaires employed in our study (UES, CSUQ, i-PANAS-SF). To measure FA, the participants were instructed to report on a 7-point Likert scale (strongly disagree; disagree; ... agree; strongly agree) their agreement to each item shown in Table 2. 
System usability. The CSUQ [32] was developed by IBM for measuring the perceived usability of systems in the context of realistic scenarios. A 7-point Likert scale of agreement (strongly agree; strongly disagree) that quantifies system usefulness is used for each of the 8 statements in the SYSUSE subscale. Two examples statements are "I am able to complete my work quickly using this search site" and "I am able to efficiently complete my work using this search site". To measure SYSUSE, the participants were instructed to report on a 7-point Likert scale (strongly disagree; disagree; ... agree; strongly agree) their agreement to each item shown in Table 3.

Custom statements. In addition to the UES and CSUQ-SYSUSE scales, we gathered information about the search sites' performance. We used a 7-point Likert scale of agreement for the following positive statements: (i) "This search site was fast in responding to my queries", (ii) "This search site helped me to accomplish my task in a reasonable amount of time", (iii) "A faster search site would help me accomplish my task quicker", and (iv) "I feel satisfied with the retrieved results". Moreover, we asked our participants to indicate on a 7-point Likert scale how frustrating each search task was.

Demographics. The study gathered the same demographics as in Section 4.1.3.

4.3.4 Participants. There were 20 participants (female $=10$, male $=10$ ) aged from 18 to 41 . The participants were of mixed nationality, came from a variety of educational backgrounds (10\% had a BSc degree, $50 \%$ had an MSc degree and $40 \%$ had a $\mathrm{PhD}$ degree), and were all proficient with the English language (10\% intermediate level, 70\% advanced level, $20 \%$ native speakers). They were primarily pursuing further studies while working $(40 \%)$ although there were a number of students (35\%) and full-time employees (25\%). Participants reported using a search site at home or work very often $(M=6.85, S E=.36)$ and also indicated that they find online searching an easy $(M=5.75, S E=1.91)$ and satisfying $(M=5.30, S E=.86)$ task. Finally, the participants were allocated randomly into two groups: one which performed the study using the slow SE (slow SE group) and another one which performed the study using the fast SE (fast SE group).

4.3.5 Procedure. The user study was carried out in a laboratory setting. At the beginning of each session the participants were informed about the conditions of the experiment and were asked to complete a demographics questionnaire and the pre-task I-PANAS-SF. Each participant then had to perform four search tasks (one for each latency value) with one of the search engines (slow SE or fast SE) they were assigned to (Section 4.3.4). The search tasks were presented in the context of a short cover story, which asked the participants to evaluate the performance of four different backend search systems. All search tasks involved submitting as many navigational queries as possible out of a list of 200 web domains, within ten minutes. Participants were presented with two web browser windows: the first window displayed the search site while the second window displayed the questionnaire. For each navigational query, participants were instructed to locate the associated URL among the first ten results of the search result page and copy-paste it in the corresponding box of the questionnaire. At the end of each search task, the participants were asked to complete the post-task I-PANAS-SF, FA, CSUQ-SYSUSE, and custom statements.

A set of training queries was used at pre-task to allow participants to familiarise themselves with the "default" behaviour of the search site and the search task. To provide further motivation and engage the participants with the search task, they were informed that a prize would be awarded to the person who will submit the most URLs in total. To control the order effects, the task assignment was randomised. Finally, the participants were randomly allocated to two search site groups, ensuring an even number of female and male participants per group.

4.3.6 Results. We present the findings based on 80 search tasks, carried out by 20 participants. For our analysis we used several related and unrelated measures tests, like the Mann-Whitney 
Table 4. Descriptive statistics (Median, SD) for reported I-PANAS-SF, FA, and CSUQ-SYSUSE scales (the reported scores were summed to obtain the final scores) for the slow SE

\begin{tabular}{|c|c|c|c|c|c|c|c|c|c|c|}
\hline \multirow[b]{2}{*}{ postPAS } & \multicolumn{2}{|c|}{$0 \mathrm{~ms}$} & \multicolumn{2}{|c|}{$750 \mathrm{~ms}$} & \multicolumn{2}{|c|}{$1250 \mathrm{~ms}$} & \multicolumn{2}{|c|}{$1750 \mathrm{~ms}$} & \multicolumn{2}{|c|}{$0-1750 \mathrm{~ms}$} \\
\hline & 17.00 & $(9.04)$ & 14.00 & (7.59) & 16.00 & $(7.21)$ & 15.00 & $(7.47)$ & 16.20 & $(7.57)$ \\
\hline postNAS & 5.00 & $(3.80)$ & 5.00 & $(2.70)$ & 6.00 & $(3.27)$ & 5.50 & $(3.28)$ & 5.50 & (3.17) \\
\hline postPAS-prePAS & -1.00 & $(8.49)$ & -2.50 & $(6.46)$ & -3.50 & $(6.34)$ & -2.50 & $(7.11)$ & -2.00 & $(6.72)$ \\
\hline postNAS-preNAS & .00 & $(2.31)$ & .00 & $(1.10)$ & 0.50 & $(1.79)$ & .00 & $(2.30)$ & .00 & $(1.76)$ \\
\hline Frustration & 2.50 & $(2.20)$ & 3.00 & $(2.02)$ & 2.00 & $(2.02)$ & 3.00 & $(2.21)$ & 2.50 & $(2.04)$ \\
\hline FA & 21.50 & $(9.37)$ & 21.00 & $(8.29)$ & 18.50 & $(9.26)$ & 21.00 & (10.38) & 21.00 & $(9.06)$ \\
\hline CSUQ-SYSUSE & 31.00 & $(6.73)$ & 29.00 & $(5.40)$ & 31.00 & (7.63) & 28.50 & $(6.89)$ & 30.00 & $(6.70)$ \\
\hline custom-1 & 5.50 & $(1.57)$ & 5.00 & $(1.55)$ & 5.00 & $(1.71)$ & 3.00 & $(1.40)$ & 5.00 & (1.59) \\
\hline custom-2 & 5.50 & $(1.18)$ & 5.00 & $(1.60)$ & 5.50 & $(1.23)$ & 4.50 & $(1.26)$ & 5.00 & $(1.34)$ \\
\hline custom-3 & 5.50 & $(1.75)$ & 6.00 & $(1.16)$ & 6.00 & $(1.90)$ & 6.00 & (1.49) & 6.00 & (1.56) \\
\hline custom-4 & 6.00 & $(.94)$ & 5.50 & $(.82)$ & 5.50 & $(1.91)$ & 5.50 & $(1.08)$ & 6.00 & $(1.28)$ \\
\hline
\end{tabular}

Table 5. Descriptive statistics (Median, SD) for reported I-PANAS-SF, FA, and CSUQ-SYSUSE scales (the reported scores were summed to obtain the final scores) for the fast $\mathrm{SE}$

\begin{tabular}{|c|c|c|c|c|c|c|c|c|c|c|}
\hline \multirow[b]{2}{*}{ postPAS } & \multicolumn{2}{|c|}{$0 \mathrm{~ms}$} & \multicolumn{2}{|c|}{$750 \mathrm{~ms}$} & \multicolumn{2}{|c|}{$1250 \mathrm{~ms}$} & \multicolumn{2}{|c|}{$1750 \mathrm{~ms}$} & \multicolumn{2}{|c|}{$0-1750 \mathrm{~ms}$} \\
\hline & 20.00 & $(7.82)$ & 18.50 & $(9.01)$ & 23.50 & $(9.48)$ & 18.50 & $(8.23)$ & 21.00 & $(8.35)$ \\
\hline postNAS & 5.50 & $(2.44)$ & 6.50 & $(3.03)$ & 6.00 & $(2.72)$ & 6.50 & (2.49) & 6.00 & $(2.58)$ \\
\hline postPAS-prePAS & 2.50 & $(5.95)$ & 1.00 & (6.13) & 1.50 & $(6.01)$ & 1.50 & $(6.29)$ & 2.00 & $(5.90)$ \\
\hline postNAS-preNAS & .00 & $(2.46)$ & .00 & $(2.53)$ & 0.50 & $(2.74)$ & .00 & $(1.33)$ & .00 & $(2.25)$ \\
\hline Frustration & 2.50 & $(1.40)$ & 3.50 & (1.63) & 3.00 & $(1.08)$ & 3.00 & $(.84)$ & 3.00 & (1.27) \\
\hline FA & 25.00 & $(10.41)$ & 26.50 & (9.23) & 27.50 & $(9.85)$ & 26.00 & $(10.56)$ & & \\
\hline CSUQ-SYSUSE & 36.50 & $(5.35)$ & 33.50 & $(8.25)$ & 31.50 & (8.34) & 35.00 & $(8.22)$ & 33.50 & (8.47) \\
\hline custom-1 & 6.00 & $(1.72)$ & 5.50 & $(1.87)$ & 4.50 & $(1.90)$ & 6.00 & $(1.64)$ & 6.00 & $(1.78)$ \\
\hline custom-2 & 6.00 & (1.18) & 5.50 & $(1.48)$ & 5.00 & $(1.23)$ & 6.00 & (1.35) & 6.00 & (1.29) \\
\hline custom-3 & 3.50 & $(2.16)$ & 5.00 & $(2.06)$ & 4.50 & $(2.23)$ & 5.00 & $(1.89)$ & 5.00 & (2.03) \\
\hline custom-4 & 6.00 & $(1.25)$ & 6.00 & $(.74)$ & 6.00 & $(1.42)$ & 6.00 & $(.95)$ & 6.00 & $(1.08)$ \\
\hline
\end{tabular}

and Wilcoxon Signed-Rank test for pair-wise comparisons, and Friedman's ANOVA for three or more conditions. Participants response to the 7-item PAS, 7-item NAS, 9-item FA, and 8-item CSUQ-SYSUSE scales were summed to obtain the final scores. Results are reported at a statistical significance level of .05. To take an appropriate control of Type I errors in multiple pair-wise comparisons we applied the Bonferroni correction.

Experienced affect. Tables 4 and 5 (top) show the median scores for the positive (postPAS) and negative (postNAS) affect scale at post-task, as well as the difference $\Delta$ s between the scores reported at pre- and post-task for the slow and fast SEs. The results indicate a decrease in positive affect for both search sites as we introduce larger fixed latency values. The inverse effect is observed for negative affect, which increases as higher fixed latency values are introduced, but this effect is more consistent in the case of the slow SE. None of the differences identified above were statistically significant. However, when comparing the reported postPAS and postNAS scores between the slow $(M d n=16.20)$ and fast $(M d n=21.00)$ SEs and across all fixed latency values, the Mann-Whitney test indicated a statistically significant difference for postPAS, $U=550.50, p<.05, r=-.31$. This small to medium effect observed for PAS between the two search sites suggests a positive bias towards 
the fast SE, despite participants having experienced the same range of fixed latency values. Tables 4 and 5 also display the median scores for reported level of frustration. There were no differences among the fixed latency values, nor between the two search sites.

Focused attention. Tables 4 and 5 (middle) display the median scores for FA. For the participants of the slow SE, the variation of the scores across the fixed latency values did not indicate any visible trend. For the participants of the fast SE, we observed a decrease in small- and medium-size fixed latency although no significant effect was established. When comparing the reported FA between the participants of the slow $(M d n=21.00)$ and fast $(M d n=26.00)$ SEs, and across all fixed latency values, the Mann-Whitney test indicated a statistically significant difference, $U=568.50, p<.05, r=-.27$ This represents a small to medium effect for the FA observed between the two search sites. Moreover, it suggests that the participants of the fast SE felt more deeply involved with the search task, despite having experienced the same range of fixed latency values.

System usability. Tables 4 and 5 (bottom) display the median CSUQ-SYSUSE scores per fixed latency value and per search site. For both search sites we observed a noticeable increase in the reported usability scores. More in specific, for the slow SE, there was a statistically significant difference in the perceived usability of the search site depending on which amount of fixed latency was introduced, $\chi^{2}(3)=11.00, p<.05$. Post-hoc analysis with Wilcoxon Signed-Rank test indicated a statistically significant difference in the perceived usability, as reported scores were significantly higher for the fixed latency value of " 0 " $(M d n=31.00)$ compared to " 1750 " $(M d n=28.50), Z=$ $-2.66, p<.008, r=-0.42$. This represents a large effect in the levels of perceived usability when fixed latency was increased by $1750 \mathrm{~ms}$. No significant differences were observed for the fast SE, suggesting that the participants were more tolerant towards the delays experienced for that search site despite the large fixed latency values introduced to their search interactions.

Additionally, the reported scores for perceived usability differed significantly between the participants of the slow $(M d n=30.00)$ and fast $(M d n=35.00)$ SEs, $U=596.00, p<.05, r=-.22$. None of the differences identified in the number of submitted queries per fixed latency value were significant. However, when comparing the number of submitted queries between the two search sites, the Mann-Whitney test revealed a statistically significant difference, $U=390.00, p<.01, r=-.44$. The large effect suggests that participants who interacted with the fast SE were able to submit more queries $(M d n=38.00)$ compared to participants who interacted with the slow SE $(M d n=49.50)$, across all queries.

Search experience. We evaluated the search experience promoted by the two search sites by asking our participants to report their agreement to a set of custom statements. With respect to statement (i), the Friedman's ANOVA test indicated for the slow SE a significant difference in the perceived search site speed, depending on which fixed latency value was added. Multiple pair-wise comparisons were performed to follow up this finding. The Wilcoxon test indicated a significant difference between the fixed latency value " 0 " $(M d n=5.50)$ and " 1750 " $(M d n=3.00)$, with "1750" receiving significantly lower scores of agreement, $T=76.5, p<.05$. Furthermore, the reported perceived search site speed by participants of the slow SE did not differ significantly from that of participants of the fast SE, which is an interesting finding considering the notable difference in the search sites' performance. In regards to statement (ii), participants' belief that the search site helped them accomplish their task more quickly changed significantly over the fixed latency values $\left(\chi^{3}=10.80, p<.05\right)$. This effect was observed only for the slow SE. Post hoc tests revealed a statistically significant difference between the fixed latency values " 0 " $(M d n=5.50)$ and "1750" $(M d n=4.50), T=74.5, p<.05$. Finally, for statements (iii) and (iv), none of the differences identified in the reported scores were statistically significant across the search sites and fixed latency values. 
Table 6. Summary of correlations of subjective beliefs on search site performance and reported UE and CSUQ-SYSUSE scales

\begin{tabular}{lcccc}
\hline & $\begin{array}{c}\text { Slow SE will respond } \\
\text { fast to my queries }\end{array}$ & $\begin{array}{c}\text { Slow SE will provide } \\
\text { relevant results }\end{array}$ & $\begin{array}{c}\text { Fast SE will respond } \\
\text { fast to my queries }\end{array}$ & $\begin{array}{c}\text { Fast SE will provide } \\
\text { relevant results }\end{array}$ \\
\hline postPAS & $.455^{* *}$ & .262 & $-.051^{* *}$ & -.272 \\
postNAS & .041 & -.083 & .245 & .133 \\
\hline FA & $.702^{* *}$ & $.720^{* *}$ & $.341^{*}$ & -.133 \\
\hline CSUQ-SYSUSE & .267 & $.411^{* *}$ & $.591^{* *}$ & $.378^{*}$ \\
\hline custom-1 & .177 & .278 & $.330^{*}$ & .212 \\
custom-2 & .177 & .263 & $.443^{* *}$ & .259 \\
custom-3 & .105 & .011 & -.182 & -.034 \\
custom-4 & .082 & .232 & $.624^{* *}$ & $.390^{*}$ \\
\hline
\end{tabular}

*. Correlation is significant at the .05 level (2-tailed). ${ }^{* *}$. Correlation is significant at the .01 level (2-tailed).

Coming back to statement (i), we further examined the participants' reported agreement (Section 4.1) to the following statements from the first study: (ii) "X search will respond fast to my queries" and (ii) "X search will provide relevant results". The Wilcoxon text revealed that participants' prior beliefs of search site speed was significantly higher $(T=25.5, p<.01)$ for the fast SE $(M d n=6.00)$ compared to the slow SE $(M d n=5.00)$. In addition, participants' prior beliefs of results relevance was significantly higher $(T=35.5, p<.05)$ for the fast SE $(M d n=6.00)$ compared to the slow SE $(M d n=5.00)$. These results help us understand that the subjective search experience may be influenced by branding, as well as users' preconceptions about the search site performance. For example, a search site perceived as "fast" or "efficient" may still result in engaging search interactions despite occasional poor performance. This suggests that a successful marketing approach could go a long way to improve the reputation of a product and bias the end-users in a positively manner.

Correlation analysis of all factors. Finally, we report the results of a correlation analysis performed across all search experience factors discussed above, including participants' prior beliefs of the search site performance. The importance of this analysis is to understand better the influence of subjective beliefs on the hedonic and cognitive aspects on the search experience. Table 6 shows all interactions between UE and CSUQ-SYSUSE factors, and subjective beliefs. We observe that in the case of the slow SE, positive bias in regards to the search site speed results in higher positive affect and FA, whereas strong belief that the search site will provide relevant results is positively correlated with perceived usability. On the other hand, for the fast SE, we observe that participants' positive expectations regarding the search site speed is negatively correlated with positive affect and positively correlated with FA and perceived usability. Moreover, this favourable bias is also positively correlated with expectations that the given search site will respond fast to the queries, will be helpful in accomplishing the task in a reasonable amount of time, and will provide satisfactory results. Despite our relatively small sample, these findings suggest that search engine bias cannot be ruled out and users tend to interpret ambiguous evidence as supporting their existing beliefs. Hence, these tendencies to overestimate, or underestimate, system performance biases their interpretations of search interactions and invokes negative behaviours that may result in search site abandonment.

\subsection{Summary}

In response to RQ2, our second user study investigated the effects of response latency on user engagement and satisfaction. The main findings of this study are summarised below. 
First, regarding the experienced affect, our analysis revealed a non-significant decrease in positive affect for both search sites as larger latency values were introduced, whereas for the negative affect we observed an increasing trend which is also not statistically significant. Moreover, the positive affect experienced at post-task was found to be significantly higher for the fast SE, indicating a potential positive bias towards the latter. These findings appear to be consistent with what is reported in [5], where the authors did not establish any significant effects of response latency on users. However, we note that in [5], the search site condition was fixed and the authors did not perform the same brand comparison across different commercial search sites as we report in the current study.

Second, with respect to FA, the reported scores of the users of the slow and fast SEs were found to be statistically significantly different. On the other hand, the reported FA within each group did not change significantly across the latency values. This finding appears to be analogous with what is reported in [5], where the FA scale revealed a pattern which, although not statistically significant, suggests a reduced engagement as the latency increases to higher values.

Third, we observed a significant difference in the perceived usability between the latency values of "0" and " 1750 " ms for the slow SE, while for the fast SE the reported usability scores did not vary significantly across the latency values. Similarly, the analysis in [5] did not indicate any significant differences in the CSUQ-SYSUSE scores across the latency values. However, when comparing the reported usability scores between the participants of the slow SE and the fast SE, we noted a significant difference (participants of the fast SE reported on average higher perceived usability), despite the participants having experienced the same latency conditions.

Last, when we examined the search experience promoted by the two search sites we noted several significant effects. More specifically, the participants of the slow SE reported a significantly slower search site speed in response to their queries, as the latency increased, while the participants of the fast SE did not suffer from such an effect. Also, the participants of the slow SE where significantly less in agreement to the statement that the search site helped them accomplish their task more quickly, as the latency values increased. No such effect was observed for the participants of the fast SE. Finally, no significant differences were identified with respect to the statements (iii) and (iv).

\section{LARGE-SCALE QUERY LOG ANALYSIS}

In this section, we investigate the impact of increasing response latency on the click behaviour of real web search engine users. To this end, we use a random sample of search queries obtained from Yahoo Web Search. We examine the variation in the click behaviour using the entire query sample as well as certain subsets of the sample with respect to one user attribute (i.e., gender, age, or level of activity), time attribute (i.e., work or non-work hours), or query attribute (i.e., query length, frequency, or type of information need). We select these attributes as they represent the typical context associated with a web search query. These attributes have impact on users' click behaviour as we show in this section and using them as signals can help to improve the accuracy of click prediction model for web search as we will show in Section 6.

\subsection{Experimental Setting}

5.1.1 Query Log. In our log data, each search query is associated with various latency values measured at different steps of the retrieval process. In all of our experiments, we rely on the end-toend (user-perceived) latency values. We limit our analysis to queries issued from desktop computers in order to reduce the potential bias due to the differences in end user devices. In addition, we limit the user space to the US, trying to reduce the variation in the network latency due to the 
geolocation of users. Furthermore, we select only queries that were issued to a particular search data centre. The resulting sample after these filtering steps contains about 30 million queries.

5.1.2 Methodology. To quantify the engagement of users with retrieved search results, we consider for each query (i) whether the user clicked on the search result page of the query or not (i.e., click status) and (ii) the number of clicks performed on the search result page (i.e., click count) We first define specific metrics that relate to click status and click count in Section 5.2 to evaluate how increasing response latency affects user click behaviour. We then compare the impact of response latency on the click behaviour of users having different attributes (Section 5.3), issuing their queries at different times of the day (Section 5.4), or issuing different types of queries (Section 5.5), with respect to click status and click count, according to the specific metrics defined for them. Details of the metrics will be provided in the corresponding sections shortly.

\subsection{Impact of Latency on Click Behaviour}

5.2.1 Impact of Latency at First Glance. We define the clicked page ratio metric as the fraction of search result pages where at least one result link is clicked by the user and observe how increasing response latency affects the metric. In this metric, higher values imply that the users interact more often with the presented search results. To increase the granularity of measurements, we group queries into buckets at every 10 millisecond latency interval and compute the clicked page ratio metric separately for each bucket, using all queries inside the bucket. Due to the confidential nature of the data, when we display this metric in the plots, we normalise the values by the maximum value observed in the plot. This should not form a concern since we are more interested in the variation of the metric rather than the absolute metric values.

Fig. 10(a) shows the variation of the clicked page ratio metric as the response latency increases. Surprisingly, we observe the presence of two separate distributions with different peaks, instead of a monotonically decreasing distribution. This result can be explained with the observation we made when discussing Fig. 2(a): The first distribution in Fig. 10(a) corresponds to queries which are served by the result cache at low response times. Most of these cached queries are navigational queries ${ }^{4}$ whose results are likely to receive at least one click. The second distribution corresponds to queries that are served by the relatively slow backend search system. These are mostly tail queries, which are less likely to result in a click on the results.

5.2.2 Effect of Result Quality. Intuitively, the quality of results has a considerably more important effect on the clicked page ratio metric than the response latency. In general, users are less likely to click on irrelevant results even if they are served with low response latency. On the other hand, if the results are expected to be very relevant, users may be willing to engage with the results, tolerating the high response latency.

One way to reduce the influence of result quality in our analysis is to group queries according to the likelihood of their results being clicked. The intuition here is that, if a query is very likely to result in a click on the search results (e.g., query "facebook"), this implies that the results are very often satisfactory for the users. In this case, any variation in the clicked page ratio is more likely to be due to the changes in user-perceived response latency. Similarly, if the results of a query rarely receive clicks, the variation in the clicked page ratio is more likely to be due to the change in latency.

Based on this intuition, we group queries into five buckets such that the clicked page ratio of all queries within a bucket fall into the same interval. According to Fig. 10(b), for every query group, the clicked page ratio tends to decrease when the response latency increases, making a bottom

\footnotetext{
${ }^{4}$ We detect navigational queries using the classifier described in Section 5.5.3.
} 


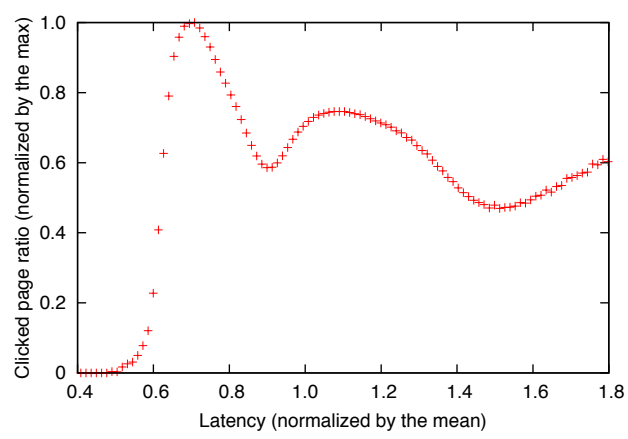

(a) All queries.

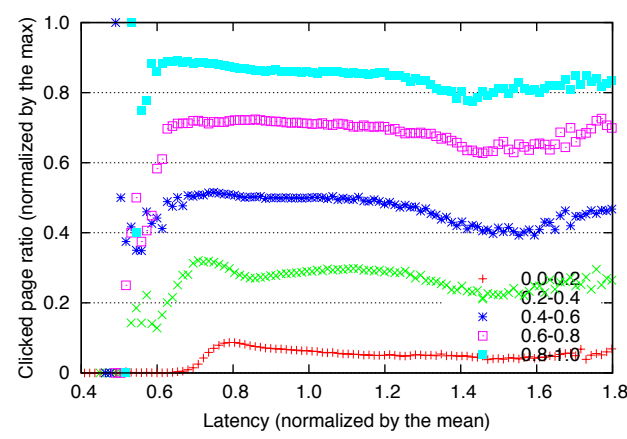

(b) Query groups w.r.t clicked page ratio.

Fig. 10. The clicked page ratio metric as latency increases.

around $1.45 \mu$. This is probably because when the response latency exceeds a tolerable value, the users simply give up the current query, submit another query, or simply switch to another task other than searching.

5.2.3 Eliminating the Effect of Result Quality. Here, we devise an evaluation method that will completely eliminate the effect of result quality in our analysis. In this method, we generate all possible pairs of queries such that the query string and retrieved search result pages are identical for the queries in a pair (the 30 million queries used in Section 5.1.1 result in 4.9 billion such pairs). We then check whether the users prefer the result set of a query in the pair over the result set of the other query. A search result set is considered to be "preferred" if it receives more clicks than the other search result set. In what follows, we refer to the query whose results were served with higher response time as the slow query, and the other query in the pair is referred to as the fast query. In this analysis, we were interested in observing the following cases:

- Click-on-fast: At least one search result of the fast query was clicked while no result of the slow query was clicked.

- Click-on-slow: At least one search result of the slow query was clicked while no result of the fast query was clicked.

- Click-more-on-fast: At least one search result is clicked for both queries, but more results are clicked in the case of the fast query.

- Click-more-on-slow: At least one search result is clicked for both queries, but more results are clicked in the case of the slow query.

We define the click-on-fast metric as the fraction of query pairs falling in the click-on-fast case, i.e., the number of pairs falling in the click-on-fast case divided by the total number of pairs (independent of the number of clicks received by fast and slow queries). The click-on-slow, click-more-on-fast, and click-more-on-slow metrics are defined in the same way for the corresponding cases described above. We define the fast-click-ratio metric as the ratio between the click-on-fast and click-on-slow cases. fast-click-ratio larger than 1 means the fast query was clicked more than the slow query while fast-click-ratio less than 1 means the opposite. We define the fast-click-more-ratio metric as the ratio between the click-more-on-fast and click-more-on-slow cases. fast-click-more-ratio larger than 1 means more search results are clicked for the fast query than for the slow query. Note that 


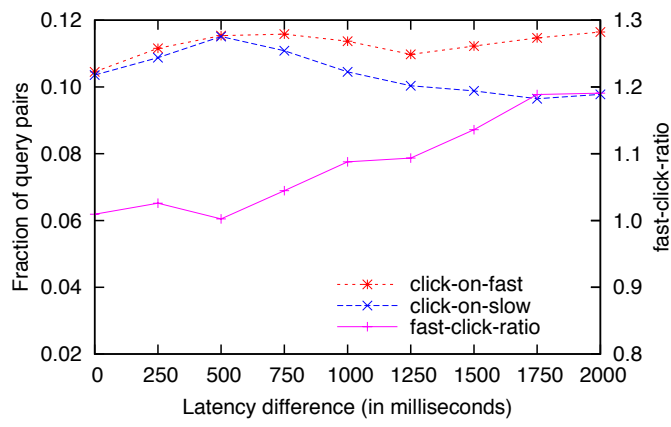

(a) Click status.

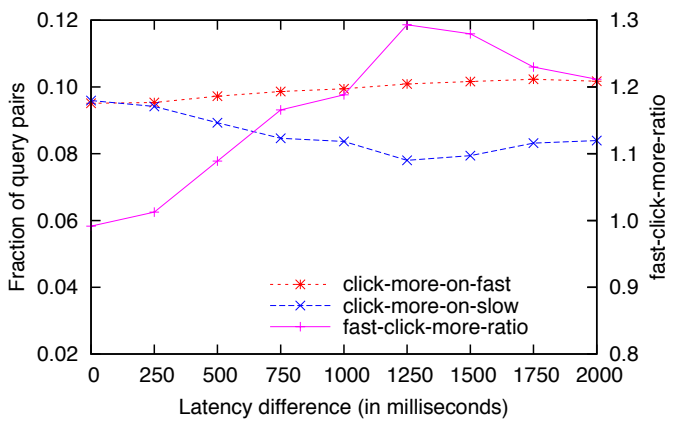

(b) Click count.

Fig. 11. Impact of latency on the click behaviour of users.

when computing the ratios, only query pairs for which the search result pages receive different number of clicks are used.

Fig. 11(a) shows click-on-fast and click-on-slow as well as fast-click-ratio for different group of query pairs. Each data point in the plot is computed using query pairs where the latency difference between the fast query and the slow query is larger than the value shown on the $\mathrm{x}$-axis, and is computed using at least 14.6 million query pairs. As an example, let us consider the $\mathrm{x}$-axis value 1000: $11 \%$ of pairs fall into the click-on-fast case (the red line in the y-axis on the left) and 10\% of pairs fall into the click-on-slow case (the blue line in the y-axis on the left). This corresponds to $79 \%$ of pairs having both the fast query and the slow query clicked or not clicked, which we do not explicitly report. According to the figure, when the latency difference increases, the value of click-on-fast increases while the value of click-on-slow decreases. The value of fast-click-ratio is always larger than one (see the red line in the y-axis on the right), implying that given two identical sets of search results (for two identical queries), users are more likely to click on a result retrieved with lower latency. This becomes more evident as the latency difference increases, confirming our observation in Fig. 10(b).

The results of a similar analysis on the click-more-on-fast, click-more-on-slow, fast-click-more-ratio metrics is shown in Fig. 11(b). In this analysis, there are 732 million query pairs, for which both search result pages are clicked. Each data point in the plot is computed using at least 1.8 million query pairs. We observe that the value of fast-click-more-ratio starts to decrease once the latency difference reaches $1250 \mathrm{~ms}$. That is, clicking on search results becomes preferable to submitting new queries due to very high response latency. This behaviour may be explained by the cost-interaction hypothesis [3].

\subsection{Experiments with Different User Attributes}

In this section, we analyse the interplay between search response latency and different user attributes, such as gender, age, and level of search activity. The age and gender information were self-reported by the users in their account settings and were available in the query log. The level of search activity is determined based on the number of queries a user issued during a day (more details are given in Section 5.3.3). As in the previous section, in order to eliminate the potential bias due to variation in result quality, we focus only on query pairs that have identical query strings 


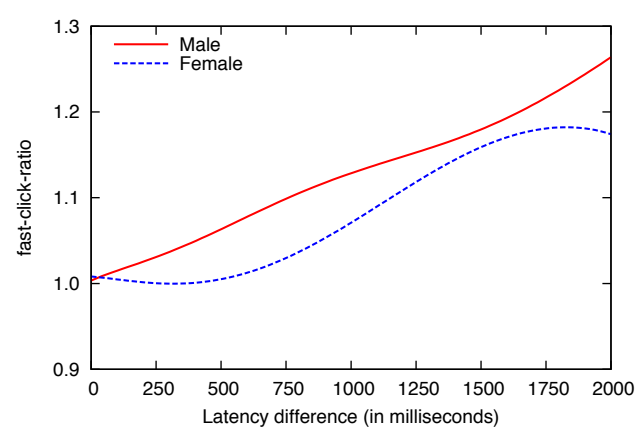

(a) Click status.

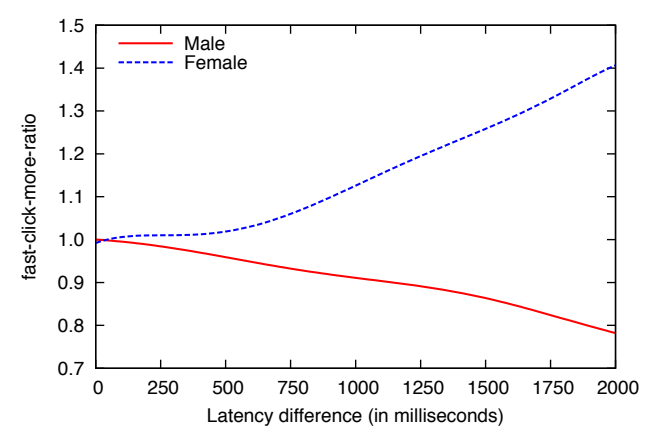

(b) Click count.

Fig. 12. Impact of different response latencies on clicks of the same query for users of the same gender.

and search results. As before, we omit a query pair if the search results of both queries in the pair received the same number of clicks.

5.3.1 Gender. Herein, we are interested in observing how the metrics introduced in Section 5.2.3 differ for male and female users. Therefore, we analyse only pairs of queries that were served with different response latencies and issued by users of the same gender. This analysis involves in over 1.5 billion pairs related to male users and female users respectively. Fig. 12 shows the fast-click-ratio (Fig. 12(a)) and the fast-click-more-ratio (Fig. 12(b)) as defined in Section 5.2.3, for query pairs belonging to male or female users. Each data point in the plot is computed using query pairs where the latency difference between the fast query and the slow query is larger than the value shown on the x-axis, representing at least 2.4 million pairs. According to Fig. 12(a), as the latency difference between the queries in a pair increases, both male and female users are more likely to click on the results of the fast query. According to Fig. 12(b), as the difference increases, female users perform a larger number of clicks on the results of the fast query. On the contrary, male users perform a larger number of clicks on the results of the slow query.

In the second experiment, we analyse pairs of queries that were served with comparable response latencies (i.e., latency difference within $250 \mathrm{~ms}$ ) and issued by users of different genders. This experiment is conducted using 1.3 billion pairs. In this experiment, we are interested in the following cases:

- Male-click: Male user clicked at least one result while female user did not click any result.

- Female-click: Female user clicked at least one result while male user did not click any result.

- Male-click-more: At least one result was clicked for both queries, but male user clicked more results.

- Female-click-more: At least one result was clicked for both queries, but female user clicked more results.

We define the male-click metric as the fraction of query pairs falling in the male-click case, i.e., the number of pairs falling in the male-click case divided by the total number of pairs (independent of the number of clicks received by the queries of the male user and the female user). The female-click, male-click-more, and female-click-more metrics are defined in the same way for the corresponding cases described above. We define the metric male-click-ratio as the ratio between the male-click and female-click cases. The values of male-click-ratio larger than 1 indicate that, for queries with 


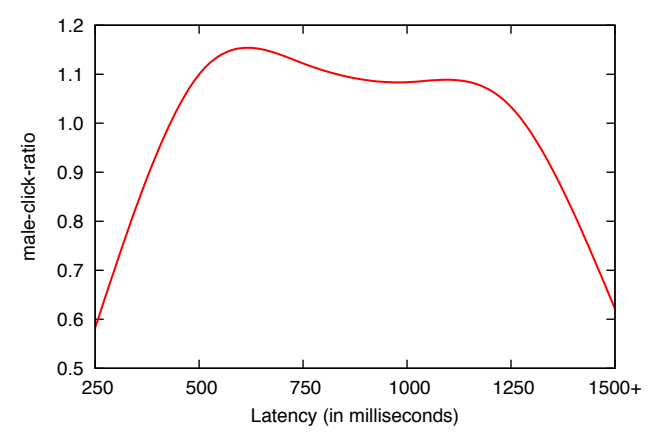

(a) Click status.

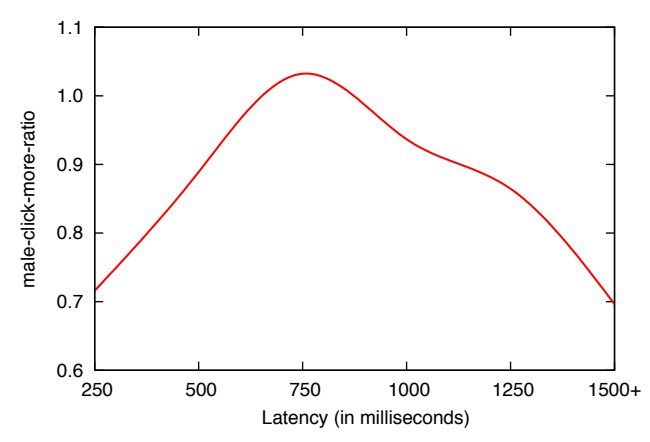

(b) Click count.

Fig. 13. Difference in clicks for users of different genders facing comparable response latencies for the same query.

comparable response latency, male users are more likely to click on search result pages than female users. We define the male-click-more-ratio metric as the ratio between the male-click-more and female-click-more cases. Values of male-click-more-ratio larger than 1 indicate that, for queries with comparable response latency, male users are likely to click more results on search result pages than female users.

Figs. 13(a) and 13(b) show male-click-ratio and male-click-more-ratio for query pairs with comparable latencies respectively. Each point is computed with at least 14.7 thousand query pairs. In Fig. 13(a), we observe that male users are more likely to click on search result pages returned with moderate latencies than female users. Similarly, in Fig. 13(b), we observe that male users are likely to issue more clicks than female users only when search results are retrieved with moderate latencies (around $750 \mathrm{~ms}$ ).

5.3.2 Age. Now we are interested in observing the impact of search response latency on users at different ages. We divide our users into four age groups: $0-24,25-44,45-64$, and $65+$. The age groups are split in such a way that each group has enough users while preserving common understanding of young, middle-aged and old people. The middle-aged users are further split into two groups as the majority of users having ages between 25 and 64 . In the first experiment, we analyse pairs of queries that were served with different response latencies and issued by users belonging to the same age group. This involves 1.8 billion query pairs. Fig. 14(a) shows the fast-click-ratio metric as defined in Section 5.2.3 for users in different age groups. Each point is computed using at least 49,200 query pairs. We observe that the ratios are always larger than one, i.e., the users in all age groups are more likely to issue a click when search results are served with lower latencies. Moreover, we observe that older users (i.e., older than 45) click on search results of the fast query with higher likelihood as the latency difference between the queries in the pair increases. On the contrary, for the young users in the 0-24 age group, when the latency difference exceeds $1000 \mathrm{~ms}$, the ratio between the number of click-on-fast and click-on-slow cases starts to decrease.

Fig. 14(b) shows the fast-click-more-ratio for users at different ages and query pairs with different latency differences. We observe that when the difference between the response latencies of the queries in a pair increases, users who are older than 45 tend to click more on the results of the slow query while users who are younger than 45 tend to click more on the results of the fast query. This 


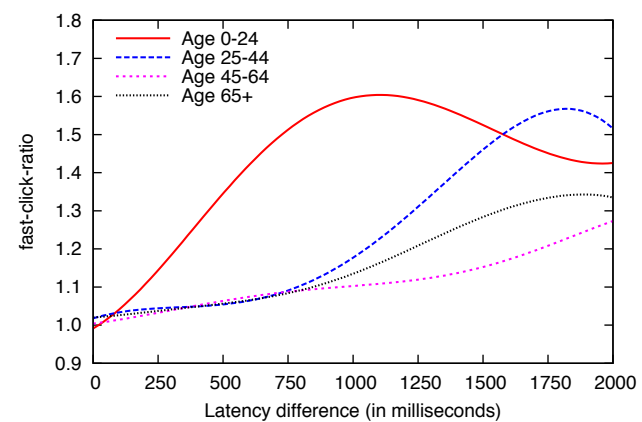

(a) Click status.

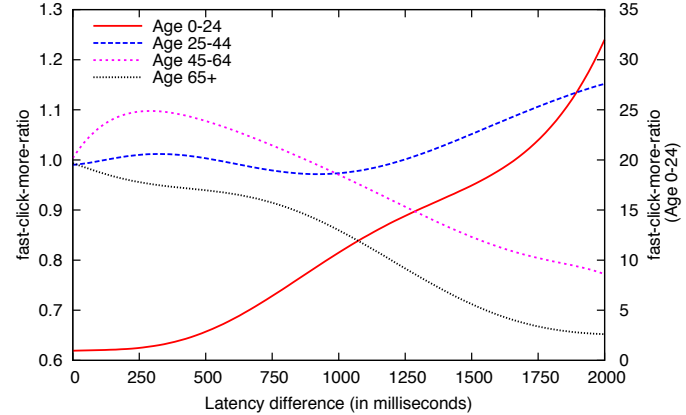

(b) Click count.

Fig. 14. Impact of different response latencies on clicks of the same query for users in the same age group.

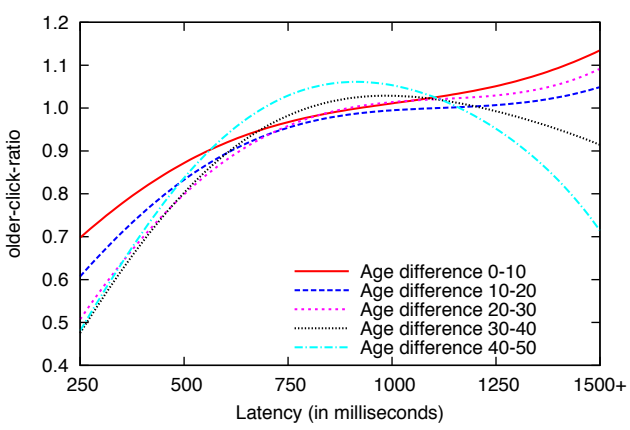

(a) Click status.

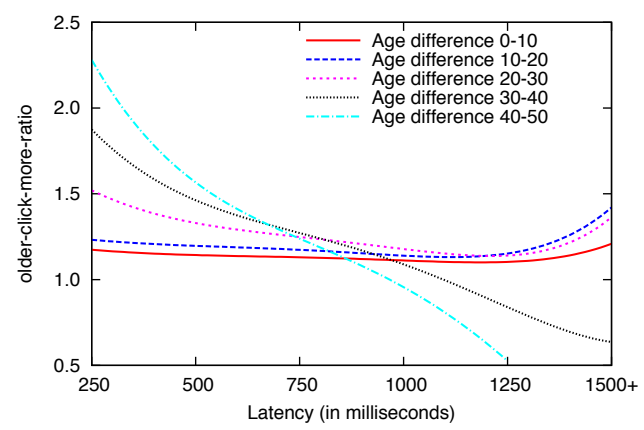

(b) Click count.

Fig. 15. Difference in clicks for users in different age groups facing comparable response latencies for the same query.

is especially true for users who are under 25 (as shown on the y axis at the right side of the plot in Fig. 14(b)).

In the second experiment, we analyse pairs of queries that were served with comparable response latencies and issued by users belonging to different age groups, accounting for 1.2 billion query pairs. Moreover, we divide the query pairs into five groups according to the difference between the ages of the users issuing the queries in the pair. In this experiment, we observe the following cases:

- Older-click: Older user clicked at least one result while younger user did not click any result.

- Younger-click: Younger user clicked at least one result while older user did not click any result.

- Older-click-more: At least one result was clicked for both queries, but older user clicked more results.

- Younger-click-more: At least one result was clicked for both queries, but younger user clicked more results. 
We define the metric older-click as the fraction of query pairs falling in the older-click case, i.e., the number of pairs falling in the older-click case divided by the total number of pairs (independent of the number of clicks received by the queries of the younger user and the older user). The younger-click, older-click-more, and younger-click-more metrics are defined in the same way for the corresponding cases described above. We define the older-click-ratio metric as the ratio between the older-click and younger-click cases. older-click-ratio larger than 1 means for queries with comparable response latency, older users are more likely to click on search result pages than younger users. We define the older-click-more-ratio metric as the ratio between the older-click-more and youngerclick-more cases. The values of older-click-more-ratio larger than 1 imply that, for queries with comparable response latency, male users are likely to click more results on search result pages than female users.

Fig. 15 shows the older-click-ratio and older-click-more-ratio metrics for the five groups of user age differences. Each point is computed with at least 9.6 thousand pairs. According to Fig. 15(a), when the response latency is low, younger users are more likely to issue a click on search results compared to older users. However, as the latency increases, older users become more likely to issue a click. This observation becomes more evident as the age difference between the users increases. We also observe from Fig. 15(b) that, when the latency is low, older users are more likely to issue a larger number of clicks on the search results.

5.3.3 Level of Search Activity. In this section, we analyse the impact of search response latency on users with varying levels of search activity. We consider a user as active if the user issues more than 10 queries during a day, on average. Otherwise, the user is considered as inactive. This threshold of activity is determined such that the query pairs with both queries issued by active users account for $10 \%$ of the query pairs with both queries issued by users at the same activity level. Note that we do not determine the threshold using a percentage of users having the highest number of queries per day due to confidentiality of the data.

In the first experiment, we analyse pairs of queries that were served with different response latencies and issued by users belonging to the same activity level. This gives us 294.2 million pairs. Fig. 16 shows the fast-click-ratio and fast-click-more-ratio metrics, as defined in Section 5.2.3, for different response latency differences and each point in the figures is computed with at least 811.2 thousand pairs. According to Fig. 16(a) where we report the fast-click-ratio, both active and inactive users are more likely to issue a click on the results of the fast query (i.e., fast-clickratio $>1$ ), especially when the latency difference between the queries is large. This observation is more pronounced for active users. In Fig. 16(b) where we report the fast-click-more-ratio, we further observe that the response latency has almost no impact on the number of results clicked by inactive users (i.e., fast-click-more-ratio is always close to 1). In contrast, when the latency difference increases, active users issue a larger number of clicks on results that are served with higher latency.

In the second experiment, we analyse pairs of queries that were served with comparable response latencies and issued by users belonging to different activity levels. This gives us 36.5 million pairs. We are interested in the following cases:

- Active-click: Active user clicked at least one result while inactive user did not click any result.

- Inactive-click: Inactive user clicked at least one result while active user did not click any result.

- Active-click-more: At least one result was clicked for both queries, but active user clicked more results.

- Inactive-click-more: At least one result was clicked for both queries, but inactive user clicked more results. 


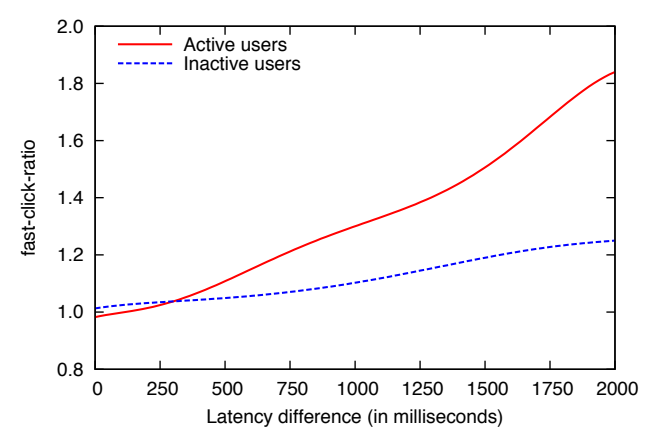

(a) Click status.

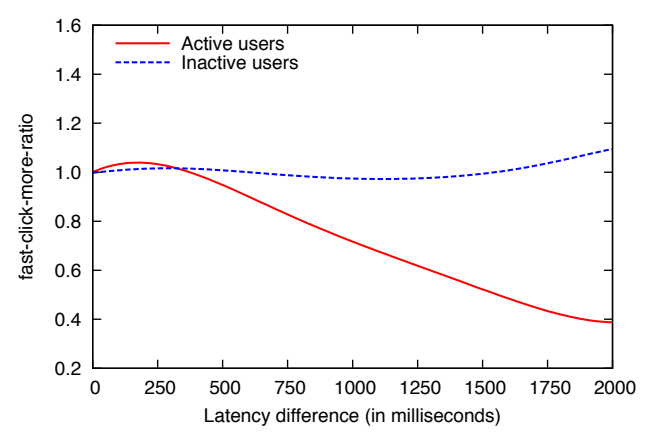

(b) Click count.

Fig. 16. Impact of different response latencies on clicks of the same query for users having the same activity level.

We define the active-click metric as the fraction of query pairs falling in the active-click case, i.e., the number of pairs falling in the active-click case divided by the total number of pairs (independent of the number of clicks received by the queries of the active user and the inactive user). The inactive-click, active-click-more, and inactive-click-more metrics are defined in the same way for the corresponding cases described above. We define the active-click-ratio metric as the ratio between the active-click and inactive-click cases. active-click-ratio larger than 1 indicates that, for queries with comparable response latency, active users are more likely to click on search result pages than inactive users. We define the active-click-more-ratio metric as the ratio between the active-clickmore and inactive-click-more cases. active-click-more-ratio larger than 1 indicates that, for queries with comparable response latency, active users are likely to click more results on search result pages than inactive users.

Fig. 17 shows the active-click-ratio and active-click-more-ratio metrics for different response latency differences and each point in the figures is computed with at least 1.9 thousand pairs. Figs. 17(a) and 17(b) shows the active-click-ratio and active-click-more-ratio respectively. We observe that, regardless of the actual latency, active users are always less likely to issue a click on the result page and issue fewer clicks than inactive users. This behaviour could be because active users are more familiar with the search engine result page and thus they can satisfy their information need simply by reading the retrieved snippets or analyzing other modules on the result page (e.g., the knowledge module), eliminating the need to issue a click on the search results.

\subsection{Experiments with Time Attribute}

We split a day into work hours (8am to 8pm) and non-work hours (8pm to 8am), and study the behavioural differences in user clicks performed within these two periods as a result of the change in response latency. We consider in this study from $8 \mathrm{am}$ to $8 \mathrm{pm}$ as work hours as most of people work from $9 \mathrm{am}$ to $5 \mathrm{pm}$ while some people may start earlier or finish later. This also allows us to have two time periods of equal length to compare with each other.

In the first experiment, we analyse pairs of queries that were served with different response latencies and issued within the same time period (i.e., work hours or non-work hours). We have 1.4 billion such pairs. Fig. 18 shows the fast-click-ratio and fast-click-more-ratio, as defined in Section 5.2.3, for different response latency differences. Each point in both figures is computed with at 


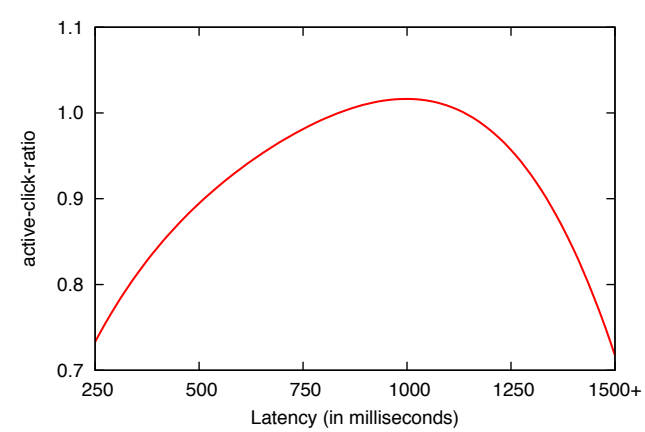

(a) Click status.

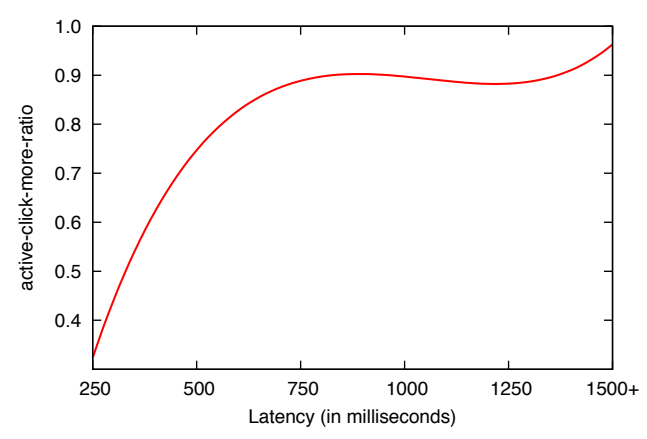

(b) Click count.

Fig. 17. Difference in clicks for users having the same activity level facing comparable response latencies for the same query.

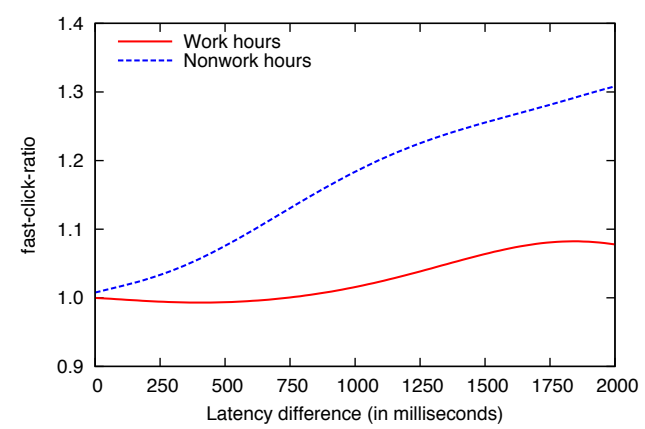

(a) Click status.

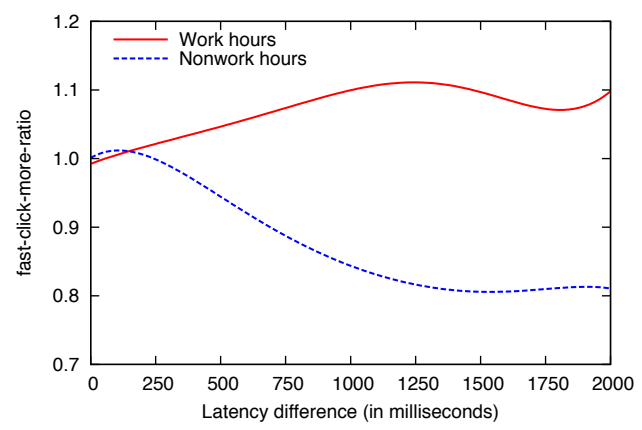

(b) Click count.

Fig. 18. Impact of different response latencies on clicks of the same query issued within the same time period.

least 2.3 million pairs. According to Fig. 18(a) that reports the fast-click-ratio, users are always more likely to issue a click on the results of the faster query, especially when the latency difference between the two queries increases and when the queries were issued in non-work hours. According to Fig. 18(b) that reports the fast-click-more-ratio, during work hours, users tend to click on more results associated with the fast query, while during non-work hours, they tend to click on more results associated with the slow query. In both cases, the click behaviour becomes more evident as the latency difference increases. When the latency difference exceeds a certain level (e.g.,1250 ms), the ratios do not change further.

In the second experiment, we analyse pairs of queries that were served with comparable response latencies but one was issued during work hours and the other was issued during non-work hours. We have 305.8 million such pairs. We are interested in the following cases:

- Work-hour-click: At least one result for the query issued in work hours was clicked while no result for the query issued in non-work hours was clicked. 


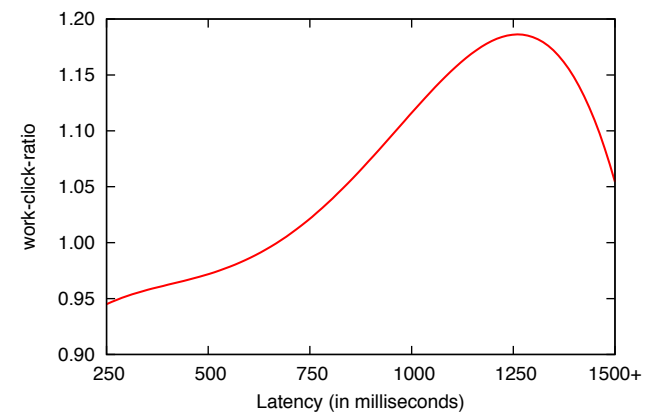

(a) Click status.

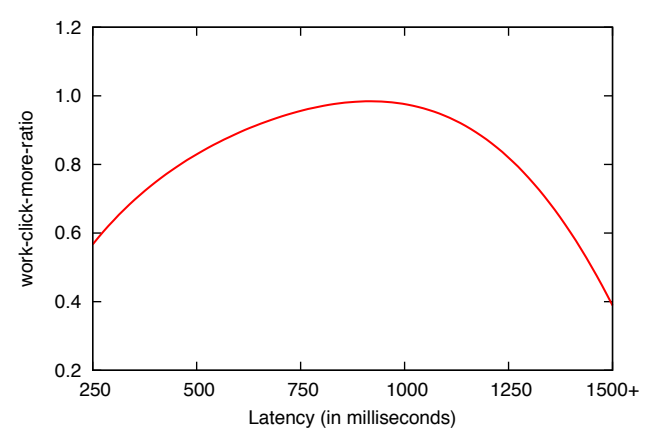

(b) Click count.

Fig. 19. Difference in clicks for the same query issued within different time periods and having comparable response latencies.

- Nonwork-hour-click: At least one result for the query issued in non-work hours was clicked while no result for the query issued in work hours was clicked.

- Work-hour-click-more: At least one result was clicked for both queries, but more results were clicked for the query issued in work hours.

- Nonwork-hour-click-more: At least one result was clicked for both queries, but more results were clicked for the query issued in non-work hours.

We define the work-click metric as the fraction of query pairs falling in the work-hour-click case, i.e., the number of pairs falling in the work-hour-click case divided by the total number of pairs (independent of the number of clicks received by the queries during work or non-work hours). The nonwork-click, work-click-more, and nonwork-click-more metrics are defined in the same way for the corresponding cases described above. We define the work-click-ratio metric as the ratio between the work-click and non-work-click cases. work-click-ratio larger than 1 indicates that for queries with comparable response latency, users are more likely to click on search result pages during work hours than during non-work hours. We also define the work-click-more-ratio metric as the ratio between the work-click-more and nonwork-click-more cases. work-click-more-ratio larger than 1 indicates that, for queries with comparable response latency, users are likely to click more results on search result pages during work hours than during non-work hours.

Fig. 19 reports the work-click-ratio and work-click-more-ratio for different response latencies. Each point in the figures are computed using at least 1.4 thousand pairs. In Fig. 19(a), we observe that, when the response latency is low enough (lower than $750 \mathrm{~ms}$ ), users are more likely to issue a click during work hours (i.e., work-click-ratio larger than 1). When the latency gets higher, users are more likely to issue a click during non-work hours. When both search result pages are clicked, users are likely to click on more results during non-work hours (i.e., work-click-more-ratio smaller than 1), as seen in Fig. 19(b).

\subsection{Experiments with Different Query Attributes}

5.5.1 Query Length. In this experiment, we analyse pairs having the same query but were served with different response latencies for queries of different lengths. We have 4.7 billion such pairs. We split queries involved in the query pairs into four groups according to their length, i.e., the number of terms in the query string (including stop words). Fig. 20 shows the corresponding distribution in 


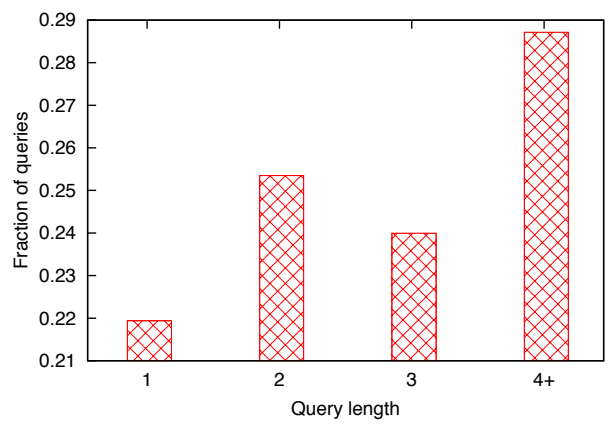

Fig. 20. Distribution of query length.

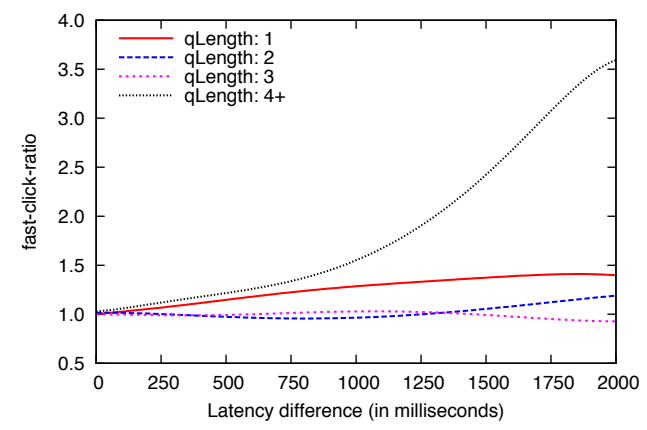

(a) Click status.

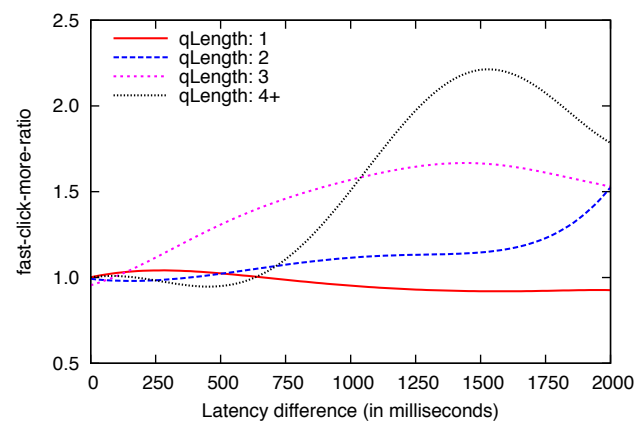

(b) Click count.

Fig. 21. Impact of different response latencies on clicks for queries with same lengths.

our sample. Fig. 21 reports the fast-click-ratio and fast-click-more-ratio, as defined in Section 5.2.3, respectively. Each point in the figures is computed using at least 2.5 million pairs. According to Fig. 21(a), when the query contains only one term or more than four terms, users are more likely to issue a click on search results returned with low latency. For queries with two or three terms and the latency differences below $1250 \mathrm{~ms}$, users do not show a clear preference towards any of the queries in the pair. When the latency difference increases, users are more likely to issue a click on the results of the fast query if the query has two terms, and on the results of the slow query if the query has three terms. According to Fig. 21(b), for queries with one term, when the latency difference increases, users are likely to issue more clicks on the results of the slow query. For longer queries, users tend to issue more clicks on the results of the fast query.

5.5.2 Query Frequency. In this experiment, we analyse pairs having the same query but were served with different response latencies for queries of different frequency. We have 4.7 billion such pairs. Fig. 22 shows the distribution of query frequency for the queries involved in the analysis We split queries into three groups according to their frequency: queries that occurred at least two times but less than five times, queries that occurred at least five times but less than 100 times, and queries that occurred more than 100 times. We note that we did not consider queries that were issued only once since such singleton queries could not be paired with any other query. 


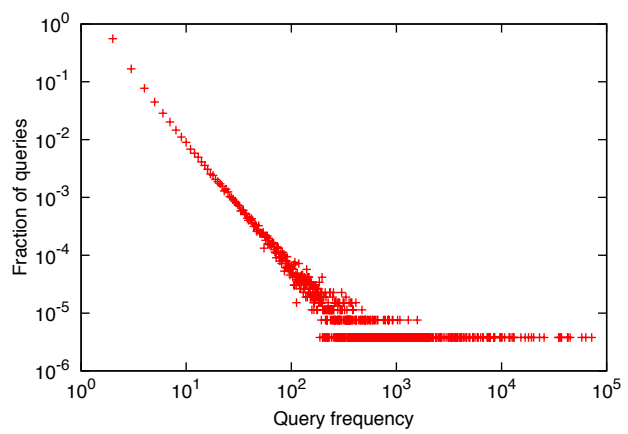

Fig. 22. Distribution of query popularity.

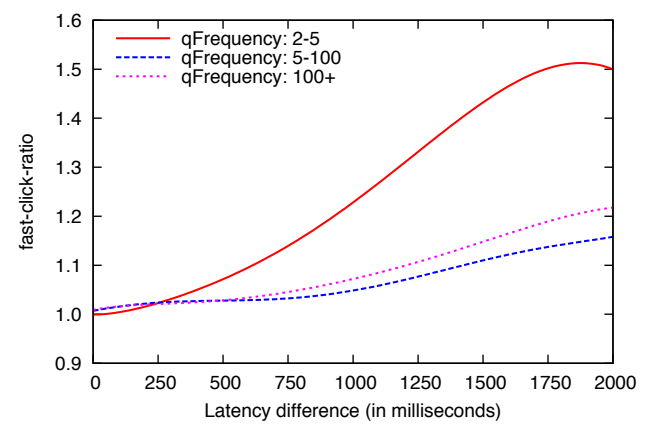

(a) Click status.

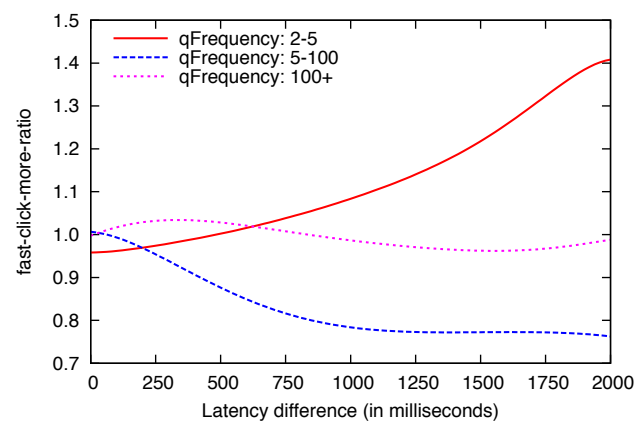

(b) Click count.

Fig. 23. Impact of different response latencies on clicks for queries within the same frequency group.

Fig. 23 reports the fast-click-ratio and fast-click-more-ratio, as defined in Section 5.2.3, respectively. Each point in the figures is computed using at least 17.5 thousand paris. In Figs. 23(a) and 23(b), we observe that, for the least frequent queries (i.e., when the query frequency is less than five), when the latency difference increases, users are more likely to click on the results of the fast query as well as clicking more results on the page. On the contrary, for highly frequent queries (i.e., when the query frequency is larger than 100), although users are also more likely to click on the results of the fast query, the gap between the click-on-fast and click-on-slow cases is smaller. In addition, users are more likely to explore more results on the search result page of the slow query when the latency difference between the two queries in a pair increases.

5.5.3 Type of Information Need. Finally, we experiment with queries representing different types of information need: navigational and informational queries. To this end, we use an in-house linear regression model that classifies each query as navigational or informational. The model was trained with queries issued on an entire day to Yahoo Web Search. The features used to train the model were mainly derived from query frequency, click frequency, and click entropy. The classification accuracy was approximately $86 \%$. Misclassified queries were mostly adult queries or queries that were difficult to classify even by human judges. Since learning an accurate query classifier was not the focus of this work, we relied on this simple classifier to label our queries. 


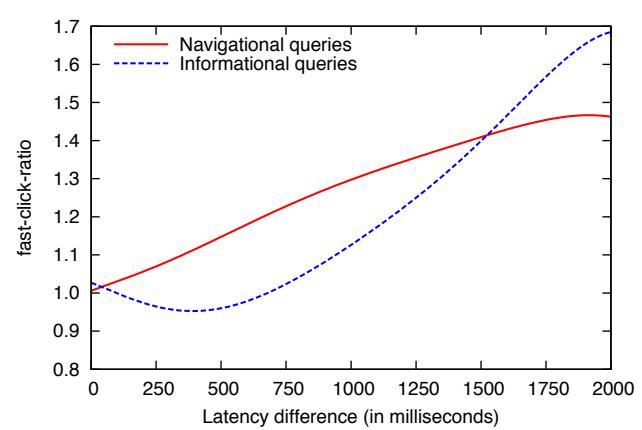

(a) Click status.

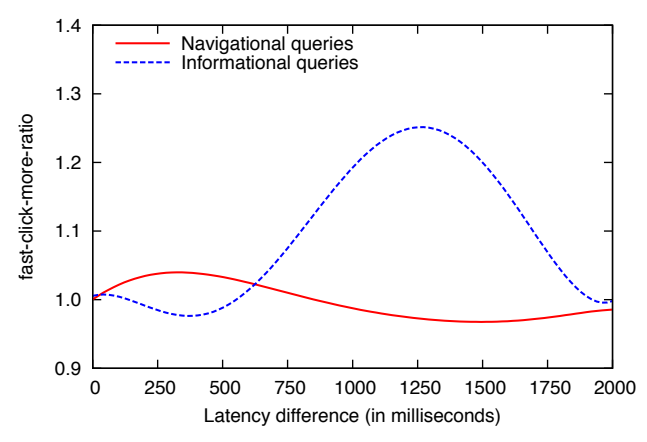

(b) Click count.

Fig. 24. Impact of different response latencies on clicks for queries having the same type of information need.

Fig. 24 shows the fast-click-ratio and fast-click-more-ratio, as defined in Section 5.2.3, for navigational and informational queries respectively. We also have 4.7 billion pairs in total for this experiment. Each point in the figures is computed using at least 23.1 thousand pairs. According to Fig. 24(a), for both types of queries, when the latency difference increases, users are more likely to issue a click on the search results of the fast query. Yet, the number of results clicked on the search results does not show much variation between the fast query and the slow query, even when the latency difference increases (Fig. 24(b)). This is not surprising since users are usually interested in a single result when they issue navigational queries. The actual latency does not affect the number of results they click on the page much if they decided to click. On the contrary, for informational queries, when the latency difference becomes high (e.g., $\geq 750 \mathrm{~ms}$ ), users are more likely to click a larger number of results on the page associated with the fast query.

\subsection{Summary}

In this section, we analysed the impact of search response latency on user click behaviour using a large-scale query-log involving billions of query pairs having identical search result pages. We showed that given two content-wise identical search result pages, users are more likely to perform clicks on the result page that is served with lower latency, and such preference is more evident when the latency difference between the two queries is larger than 500 milliseconds. This confirms our observation in Section 4.1 in that users can hardly distinguish a delayed response with up to $500 \mathrm{~ms}$ added delay and a regular response latency with no added delay for the same query, while they are likely to notice the presence of added delays higher than $1000 \mathrm{~ms}$. This also explains why we observed, in our query log analysis, different click behaviour when latency differences increase.

Our analysis also showed to which extent user attributes (i.e., gender, age, level of activity), time attribute (i.e., work and non-work hours), and query attributes (i.e., length, frequency, information need) affect users' click behaviour. We observe that, in general, female users, elder users, and inactive users can tolerate high latency better. Interestingly, although we showed in Section 4.1 that female users are more likely to react to a small change in latency than male users, this does not change their click behaviour as much as that of male users. This may be because female, elder and inactive users are likely to be less experienced than male, young and active users for web search, which makes them less sensitive to latency changes. We also showed that search response latency has lower impact on user click behaviour during work hours. This may be related to the specific 
tasks users need to accomplish for their work with certain time constraints while during non-work hours they have more freedom to abandon a search session if the search result page is returned under high latency, or explore deeper in the search result page when it contains relevant results even if it is slower. Finally, we observe that users have better tolerance on response latency for long queries, infrequent queries and, informational queries. This may be because such queries are usually more difficult to answer. Therefore, most users are still willing to spend time and issue clicks to receive relevant information even if their search results are returned with higher latency.

Our query log analysis investigated the effect of response latency on the click behaviour of users in the short term, while [42] reported results about the change in query volume in the long term. Hence, the results of the two works are not directly comparable. [46] reported a decrease in the likelihood that the users will tend to click less on a search result as response latency increases. In this respect, the main finding of our query log analysis is consistent with [46]. However, unlike [46], we observed that, if the response latency increases too much, the users will tend to click more on the result pages because they prefer to submit new queries to the search engine. In the next section, we show how the findings in this section can be leveraged to help predicting user click behaviour in web search.

\section{LEVERAGING RESPONSE LATENCY TO PREDICT CLICK BEHAVIOUR}

As we have demonstrated in previous sections, users exhibit a high variation in the way they perceive the response latency of a web search engine, depending on user attributes, temporal context, query attributes, and potentially various other parameters. In this section, we devise a machine learning framework that exploits such attributes, together with certain latency features, to predict whether a user will issue any click on the retrieved web search results or not. We note that our objective here is not to create a full-fledged click prediction framework using an extensive set of features, but rather to provide evidence on the utility of response latency in predicting user click behaviour in web search.

\subsection{Experimental Setting}

We pose the click prediction problem as a binary classification problem where the classification target is whether the user will issue a click on the retrieved web search results (isClicked) or not (isNotClicked), when exposed to a certain search response latency. To this end, we use a large, random sample of queries obtained from Yahoo Web Search. We place each query in a latency bin according to its latency value. The latency bins are created in increments of $250 \mathrm{~ms}$ (we also maintain a bin that contains all queries). We opt for this design because we anticipate a variation in user click behaviour depending on the experienced search response latency, as demonstrated by our earlier analyses in Sections 4 and 5. By modelling the click behaviour for different response latency ranges, we aim to improve our models' accuracy. Table 7 shows the distribution of isClicked and isNotClicked classes, for different response latency bins. The class sizes exhibit an imbalance of approximately $70 \%-30 \%$, isClicked being the larger class.

For our predictive modelling task, we train a Random Forest classifier ${ }^{5}$ for each latency bin using the features shown in Table 8. Prior to that, we perform feature selection using a wrapper method that evaluates multiple models using procedures that add and remove features, until finding an optimal combination that maximises the model's performance. More specifically, we use a recursive feature elimination algorithm ${ }^{6}$ that fits all predictors, ranks each predictor according to its importance, and, finally, retains the top-ranked predictors which are refitted to the model

\footnotetext{
${ }_{5}^{5}$ https://cran.r-project.org/web/packages/randomForest/index.html

${ }^{6}$ https://cran.r-project.org/package $=$ caret
} 
Table 7. Class distribution within different response latency bins

\begin{tabular}{lrrrrrrrrr}
\hline & \multicolumn{7}{c}{ Latency range $(\mathrm{ms})$} \\
\cline { 2 - 9 } Class & $\geq 0$ & $250-500$ & $500-750$ & $750-1000$ & $1000-1250$ & $1250-1500$ & $1500-1750$ & $1750-2000$ & $\geq 2000$ \\
\hline isClicked & $72.37 \%$ & $70.18 \%$ & $68.42 \%$ & $79.60 \%$ & $76.69 \%$ & $75.31 \%$ & $74.15 \%$ & $73.87 \%$ & $68.04 \%$ \\
isNotClicked & $27.63 \%$ & $29.82 \%$ & $31.58 \%$ & $20.40 \%$ & $23.31 \%$ & $24.69 \%$ & $25.85 \%$ & $26.13 \%$ & $31.96 \%$ \\
\hline
\end{tabular}

Table 8. Features used for modelling click behaviour

\begin{tabular}{lrr}
\hline Feature & Type & Range of values $(M d n)$ \\
\hline$f_{1}:$ page_load_time $(\mathrm{ms})$ & Numeric & $344-209,517(700)$ \\
$f_{2}:$ search_engine_latency $(\mathrm{ms})$ & Numeric & $136-5148(408)$ \\
$f_{3}:$ network_latency $(\mathrm{ms})$ & Numeric & $0-4681(9)$ \\
$f_{4}:$ browser_latency (ms) & Numeric & $133-209,166(274)$ \\
$f_{5}:$ user_gender & Categorical & $\{$ male, female\} \\
$f_{6}:$ user_age & Numeric & $0-100(40)$ \\
$f_{7}:$ is_work_hour & Boolean & $\{$ true, false \\
$f_{8}:$ query_length & Numeric & $1-56(2)$ \\
$f_{9}:$ query_popularity & Numeric & $1-215,162(6)$ \\
$f_{10}:$ is_navigational & Boolean & $\{$ true, false \\
\hline
\end{tabular}

Table 9. Feature ranking (according to the AUC metric) for the different latency bins

\begin{tabular}{ccccccccc}
\hline 0 & $250-500$ & $500-750$ & $750-1000$ & $1000-1250$ & $1250-1500$ & $1500-1750$ & $1750-2000$ & $\geq 2000$ \\
\hline$f_{9}: 80.47$ & $f_{9}: 82.43$ & $f_{9}: 75.87$ & $f_{9}: 82.38$ & $f_{9}: 81.35$ & $f_{9}: 82.31$ & $f_{9}: 82.31$ & $f_{9}: 82.65$ & $f_{9}: 81.25$ \\
$f_{6}: 80.84$ & $f_{3}: 83.45$ & $f_{6}: 80.94$ & $f_{6}: 85.74$ & $f_{6}: 85.15$ & $f_{8}: 83.99$ & $f_{6}: 83.47$ & $f_{8}: 83.08$ & $f_{3}: 85.24$ \\
$f_{3}: 83.60$ & $f_{6}: 86.89$ & $f_{3}: 82.15$ & $f_{4}: 88.67$ & $f_{4}: 87.65$ & $f_{4}: 85.70$ & $f_{8}: 84.55$ & $f_{4}: 86.08$ & $f_{6}: 88.32$ \\
$f_{4}: 89.16$ & $f_{2}: 92.47$ & $f_{4}: 88.61$ & $f_{8}: 90.89$ & $f_{8}: 90.31$ & $f_{6}: 90.08$ & $f_{4}: 89.66$ & $f_{2}: 89.51$ & $f_{4}: 90.93$ \\
$f_{2}: 90.97$ & $f_{4}: 93.16$ & $f_{2}: 91.09$ & $f_{3}: 91.99$ & $f_{1}: 91.60$ & $f_{1}: 91.44$ & $f_{1}: 90.82$ & $f_{1}: 90.43$ & $f_{1}: 91.65$ \\
$f_{1}: 91.16$ & $f_{1}: 93.15$ & $f_{1}: 91.42$ & $f_{2}: 92.93$ & $f_{2}: 92.09$ & $f_{3}: 92.37$ & $f_{3}: 91.63$ & $f_{6}: 91.44$ & $f_{8}: 92.30$ \\
$f_{8}: 91.21$ & $f_{5}: 92.98$ & $f_{8}: 91.70$ & $f_{1}: 93.15$ & $f_{3}: 92.63$ & $f_{2}: 92.59$ & $f_{2}: 91.72$ & $f_{3}: 91.71$ & $f_{2}: 92.55$ \\
$f_{5}: 91.45$ & $f_{8}: 93.32$ & $f_{5}: 91.32$ & & & & & & \\
$f_{7}: 91.51$ & $f_{7}: 93.45$ & $f_{7}: 91.74$ & & & & & & \\
\hline
\end{tabular}

in the next iteration. To get performance estimates that incorporate the variation due to feature selection, we apply 10-fold cross-validation. As the performance measure to optimise, we look at the Area Under Curve (AUC) of the Receiver Operating Characteristic (ROC) curve, which considers the proportion of two single-column-based evaluation metrics, namely the true positive (TP) rate and false positive (FP) rate. By applying this step, we exclude noisy features that do not contribute much to the accurate discrimination of the two targeted classes. Table 9 shows the set of features picked in the final model for each latency bin in decreasing order of their importance. The take away message here is that the latency component features (e.g., page load time, search engine latency, network latency, and browser latency) have been retained in all feature subsets as important predictors. Furthermore, demographic features like the user age and gender appear to also play a role in predicting click behaviour as well. More specifically, the user gender feature appears in all latency bins whereas the user age is selected among the informative features only for latency ranges bellow $750 \mathrm{~ms}$. It may be that user age is a determining factor for the fixed latency effect mainly for smaller latency values; for the larger latency values potentially the fixed latency impact is uniform across all age groups. 
We perform 10-fold cross-validation, using stratified sampling, to create balanced splits of the data that preserve the overall class distribution. In each fold, we retain $90 \%$ of our data for training and $10 \%$ for testing. We also held out a small subset of our training data for fine-tuning the classifier's hyper-parameters (e.g., $\epsilon$-threshold, number of trees, minimum size of terminal nodes, maximum number of terminal nodes) and apply the optimal parameter values to our final model. To deal with imbalanced training sets using the random forest algorithm, several approaches are possible like the Balanced Random Forest (BRF), Weighted Random Forest (WRF), One-side Sampling, SHRINK, and SMOTE. We opt for the synthetic minority oversampling technique (SMOTE), which is a powerful method that has proven successful in various applications [12].

The SMOTE algorithm creates artificial data based on the feature space similarities between existing minority examples. More specifically, for subset $S_{\text {min }} \in S$, we consider the $K$-nearest neighbours for each example $x_{i} \in S_{\text {min }}$. The $K$-nearest neighbours are defined as the $K$ elements of $S_{\min }$ whose euclidian distance between itself and $x_{i}$ exhibits the smallest magnitude along the $\mathrm{n}$-dimensions of the feature space $X$. To generate a synthetic sample, the algorithm selects in random one of the $K$-nearest neighbours and multiplies the corresponding feature vector difference with a random number between $[0,1]$, and, finally, adds this vector to $x_{i}$

$$
x_{\text {new }}=x_{i}+\left(\hat{x}_{i}-x_{i}\right) \times \delta
$$

where $x_{i} \in S_{\text {min }}$ is the minority instance under consideration, $\hat{x}_{i}$ is one of the $K$-nearest neighbours for $x_{i}: \hat{x}_{i} \in S_{\text {min }}$, and $\delta \in[0,1]$ is a random number. As a result, the synthetic instance according to 1 is a point along the line segment joining $x_{i}$ under consideration and the randomly selected $K$-nearest neighbour $\hat{x}_{i}$. In our case, we set the number of $K$-nearest neighbours to $K=5$. In addition to oversampling the minority class, we downsample the majority class using random selection.

Finally, we assess the performance of our final model against the test set in each fold. We note that we do not manipulate the class instances of the test set, which retains the original class distribution. For our evaluation we consider the standard IR metrics of Precision, Recall, and Accuracy. Traditionally, the most frequently used metrics are Accuracy and Error Rate. However, metrics such as Accuracy can be deceiving in certain situations and are highly sensitive to changes in data. Therefore, we also compute the F-Measure, which combines Precision and Recall as a measure of the effectiveness of classification in terms of a ratio of the weighted importance on either Recall or Precision, as determined by the $\beta$ coefficient. Finally, we compute the AUC metric.

\subsection{Experimental Results}

Table 10 provides a detailed overview of our model's micro-average performance over the ten folds, with respect to the selected metrics, and across the different response latency bins. The first set of rows shows the Precision, Recall and F-Measure for the positive class (majority class), the second set of rows shows the same metrics for the negative class (minority class), and the last set of rows shows the the weighted average version of them. In the last row, the table shows the AUC which we choose as our primary performance indicator since it is more suitable for data with imbalanced classes. Moreover, the AUC is not a function of the cutoff score used by the model; it is rather an evaluation of its performance as the cutoff score varies over all possible values.

We compare our model against a Baseline (shown in Table 11) that always predicts the majority class. We apply the Mann Witney U test for stochastic dominance to determine the cases where our model performs significantly better than the Baseline (indicated with * in Table 10) and provide the corresponding $U$ statistic. To this end, we apply a post-hoc analysis involving multiple pairwise 
Table 10. Performance of the Random Forest classifier across different latency bins; scores in parentheses are Mann-Whitney's $U$ statistic

\begin{tabular}{|c|c|c|c|c|c|c|c|c|c|}
\hline \multirow[b]{2}{*}{ Metric } & \multicolumn{9}{|c|}{ Latency bin (ms) } \\
\hline & $\geq 0$ & $250-500$ & $500-750$ & $750-1000$ & $1000-1250$ & $1250-1500$ & $1500-1750$ & $1750-2000$ & $\geq 2000$ \\
\hline \multicolumn{10}{|l|}{ Pos. class } \\
\hline Precision $(\%)$ & $86.64(100)^{\text {**** }}$ & $80.55(100)^{* * *}$ & $80.01(100)^{* * *}$ & $93.54(100)^{* * * *}$ & $91.56(100)^{* * *}$ & $79.72(100)^{* * * *}$ & $78.55(100)^{* * * *}$ & $80.13(100)^{* * *}$ & $76.82(100)^{* * *}$ \\
\hline Recall $(\%)$ & 59.04 & 94.76 & 49.25 & 73.53 & 69.02 & 90.35 & 89.26 & 87.78 & 80.18 \\
\hline F-Measure (\%) & 70.10 & $87.07(100)^{* * *}$ & 60.89 & 82.32 & 78.69 & 84.66 & 83.52 & 83.60 & 78.31 \\
\hline \multicolumn{10}{|l|}{ Neg. class } \\
\hline Precision $(\%)$ & $41.51(100)^{* * *}$ & $79.32(100)^{* * * *}$ & $40.08(100)^{* * *}$ & $43.60(100)^{* * *}$ & $43.69(100)^{* * * *}$ & $49.89(100)^{* * *}$ & $49.29(100)^{* * *}$ & $52.41(100)^{* * *}$ & $53.50(100)^{* * *}$ \\
\hline Recall (\%) & $75.90(100)^{\text {**** }}$ & $46.13(100)^{* * * *}$ & $73.29(100)^{* * *}$ & $80.08(100)^{* * * *}$ & $79.03(100)^{\text {**** }}$ & $29.58(100)^{* * *}$ & $29.58(100)^{* * *}$ & $36.81(100)^{* * *}$ & $47.87(100)^{* * *}$ \\
\hline F-Measure $(\%)$ & $53.58(100)^{* * *}$ & $58.14(100)^{* * * *}$ & $51.78(100)^{* * *}$ & $56.44(100)^{* * *}$ & $56.25(100)^{* * * *}$ & $36.60(100)^{* * *}$ & $36.39(100)^{* * *}$ & $41.78(100)^{* * *}$ & $49.85(100)^{* * *}$ \\
\hline \multicolumn{10}{|l|}{ Weighted. avg. } \\
\hline Precision $(\%)$ & $74.18(100)^{* * * *}$ & $80.18(100)^{* * *}$ & $67.38(100)^{* * *}$ & $83.38(100)^{* * * *}$ & $80.41(100)^{* * *}$ & $72.36(100)^{* * * *}$ & $71.36(100)^{* * *}$ & $72.97(100)^{* * *}$ & $69.37(100)^{* * *}$ \\
\hline Recall (\%) & 63.70 & $80.25(100)^{* * * *}$ & 56.86 & 74.86 & 71.35 & 75.35 & 73.89 & 74.62 & 69.86 \\
\hline F-Measure (\%) & $\mathbf{6 5 . 5 4}(100)^{* * * *}$ & $78.44(100)^{* * * *}$ & $58.00(100)^{* * *}$ & $77.06(100)^{* * * *}$ & $73.46(100)^{* * * *}$ & $72.80(100)^{* * * *}$ & $71.67(100)^{* * *}$ & $72.80(100)^{* * *}$ & $69.22(100)^{\text {*** }}$ \\
\hline Accuracy $(\%)$ & 63.70 & $80.25(100)^{* * * *}$ & 56.86 & 74.86 & 71.35 & 75.35 & 73.89 & 74.62 & 69.86 \\
\hline AUC $(\%)$ & 73.54 & 78.26 & 66.10 & 82.03 & 79.26 & 76.94 & 74.53 & 74.69 & 74.14 \\
\hline
\end{tabular}

Table 11. Performance of the Baseline model across different latency bins

\begin{tabular}{|c|c|c|c|c|c|c|c|c|c|}
\hline \multirow[b]{2}{*}{ Metric } & \multicolumn{9}{|c|}{ Latency bin (ms) } \\
\hline & $\geq 0$ & $250-500$ & $500-750$ & $750-1000$ & $1000-1250$ & $1250-1500$ & $1500-1750$ & $1750-2000$ & $\geq 2000$ \\
\hline \multicolumn{10}{|l|}{ Pos. class } \\
\hline Precision $(\%)$ & 72.40 & 70.16 & 68.37 & 79.66 & 76.69 & 75.33 & 74.24 & 74.18 & 68.06 \\
\hline Recall (\%) & 100.00 & 100.00 & 100.00 & 100.00 & 100.00 & 100.00 & 100.00 & 100.00 & 100.00 \\
\hline F-Measure (\%) & 83.99 & 82.47 & 81.21 & 88.68 & 86.81 & 85.93 & 85.21 & 85.17 & 81.00 \\
\hline \multicolumn{10}{|l|}{ Neg. class } \\
\hline Precision (\%) & .00 & .00 & .00 & .00 & .00 & .00 & .00 & .00 & .00 \\
\hline Recall (\%) & .00 & .00 & .00 & .00 & .00 & .00 & .00 & .00 & .00 \\
\hline F-Measure $(\%)$ & .00 & .00 & .00 & .00 & .00 & .00 & .00 & .00 & .00 \\
\hline \multicolumn{10}{|l|}{ Weighted. avg. } \\
\hline Precision $(\%)$ & 52.41 & 49.23 & 46.74 & 63.45 & 58.81 & 56.74 & 55.11 & 55.02 & 46.32 \\
\hline Recall (\%) & 72.40 & 70.16 & 68.37 & 79.66 & 76.69 & 75.33 & 74.24 & 74.18 & 68.06 \\
\hline F-Measure (\%) & 60.81 & 57.86 & 55.52 & 70.64 & 66.57 & 64.73 & 63.26 & 63.18 & 55.13 \\
\hline Accuracy $(\%)$ & 72.40 & 70.16 & 68.37 & 79.66 & 76.69 & 75.33 & 74.24 & 74.18 & 68.06 \\
\hline
\end{tabular}

comparisons, for which we correct the level of significance to control the false discovery rate by using the Benjamini-Hochberg correction [7].

At first blush, when comparing the performance data shown in Tables 10 and 11, it appears that the Baseline is doing a better job at predicting the majority class (with respect to Recall and F-Measure), and, overall, exhibits a better weighted average Recall and Accuracy. However, as noted earlier, Accuracy is a highly sensitive metric to imbalanced classes and, in such contexts, it is not as reliable as other classification performance indicators. Therefore, if we turn our attention to the weighted average F-Measure, which accounts for the TP, FP and FN cases, we observe that our model predicts the targeted classes significantly better than the Baseline for all latency bins. In addition, the Baseline fails to predict the minority class, which is paramount to applying the energy consumption optimising framework, as we discuss in Section 7.

Moving on to the AUC metric (Fig. 25), the best performance (AUC 82.03\%) is observed for the 750-1000 ms latency bin while the worst performance (AUC 66.10\%) is observed for the $500-750 \mathrm{~ms}$ latency bin. For all other latency bins, our model maintains a somewhat uniform performance that falls within the $73.54 \%-79.26 \%$ range. The performance drop observed for the $500-750$ ms latency 
bin may be due to the inherent noise of the respective data. As our analysis has indicated, users are highly likely to notice (and be affected by) the presence of delays when the delays are larger than $1000 \mathrm{~ms}$, whereas delays under $500 \mathrm{~ms}$ are not easily perceivable (see Section 4). This suggests that there is a latency window between $500 \mathrm{~ms}$ and $1000 \mathrm{~ms}$, where the response latency becomes gradually noticeable, but it is also subject to the large variation in users' ability to perceive it (Figs. 8 and 9). As a consequence, the prediction of users' click behaviour becomes even more challenging for the particular latency bin.

Another interesting finding is that our prediction accuracy improves when we trained our models using individual latency bins rather than the whole query sample (the $\geq 0 \mathrm{~ms}$ bin). We note that, for the $\geq 0 \mathrm{~ms}$ bin, our model achieves an AUC value of $73.54 \%$, which is not a truly representative performance given that AUC values observed for all other response latency bins exhibit a large variation (values are in the $66 \%-82 \%$ range). By allocating queries into relatively homogeneous bins (analogous to strata in stratified sampling), we reduce the noise and compute more reliable estimates of our models' performance. We argue that the observed variation indicates an interaction between the range of response latency and click behaviour, which we are able to learn more accurately through our design. Furthermore, we observe that our models perform best for the response latency bins of 250-500 ms and 750-1500 ms. These bins are of particular interest since they represent the early- and mid-stage latencies encountered in today's web search engines. This can be exploited to determine which query processing mechanism to apply and towards which goal (e.g., using less hardware resources, prioritising time-critical queries, etc.). Finally, considering the existing conventions for interpreting AUC values, the reported models performances suggest that we can exploit prior knowledge about the user-perceived response latency to infer users' click behaviour (RQ6), despite the somewhat simple feature set we used and the challenging nature of the click prediction problem.

The framework we introduced in this section for click prediction may be useful in different scenarios related to search efficiency. For example, if we can accurately predict beyond what point a user is not likely to click on a search result, we can schedule execution of queries [34] in such a way that queries issued by users whose search experience is more likely to be affected by high latency are processed earlier than the others. As another example, we may terminate processing of queries as soon as we predict that the user will not issue any click on the search results due to high response latency. Such an optimisation may lead to energy savings for the search engine as in the use case we will demonstrate in the next section.

\section{USE CASE: REDUCING THE ENERGY CONSUMPTION OF WEB SEARCH ENGINES}

\subsection{Motivation}

In this section, we address the research question RQ7 by presenting a use case where the click prediction model introduced in the previous section is leveraged to reduce the energy consumption of a web search engine. The basic idea behind this optimisation is to terminate the processing of a query early and present the user some partially computed search results, instead of the fully computed results, if the computational of the query takes too much time and the user engagement is predicted to be affected due to high response latency. ${ }^{7}$ That is, in this technique, the search engine prefers serving lower quality results with reasonable latency to serving its best quality results with intolerable latency (i.e., when the user-perceived latency is expected to have an impact on user engagement).

\footnotetext{
${ }^{7}$ We assume that the quality of the search results are improved in an incremental fashion as more postings are processed and relevance scores are computed for more documents. We also assume that the clicks on search results positively correlate with user engagement.
} 


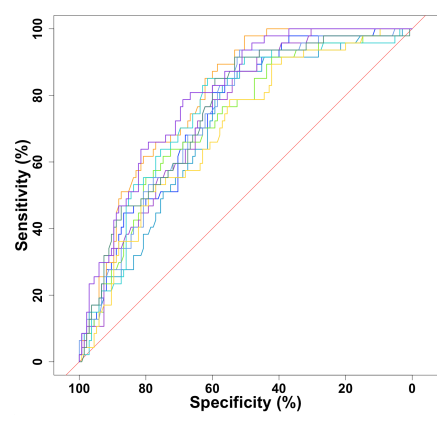

(a) $\geq 0 \mathrm{~ms}$ (AUC: $73.54 \%$ )

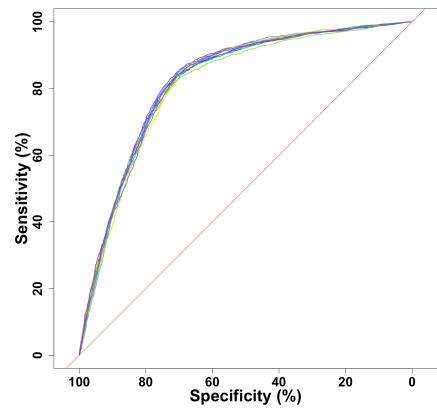

(d) 750-1000 ms (AUC: $82.03 \%$ )

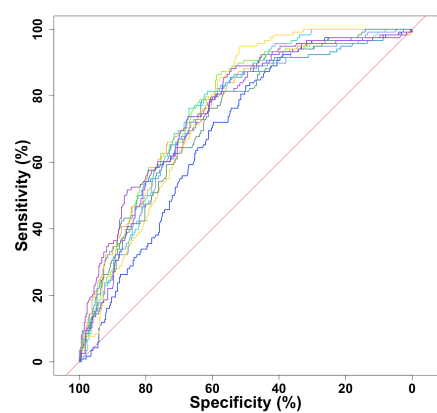

(g) 1500-1750 ms (AUC: $74.53 \%$ )

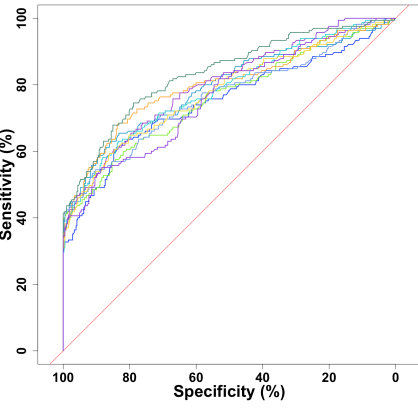

(b) $250-500 \mathrm{~ms}$ (AUC: $78.26 \%$ )

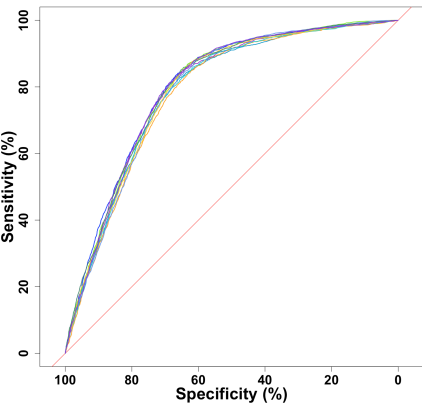

(e) 1000-1250 ms (AUC: $79.26 \%$ )

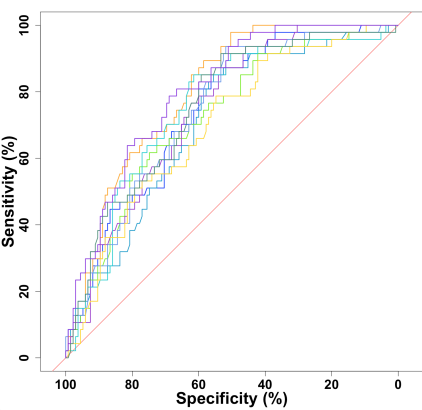

(h) 1750-2000 ms (AUC: $74.69 \%$ )

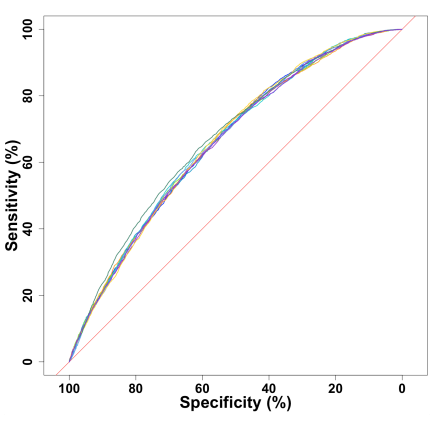

(c) $500-750 \mathrm{~ms}$ (AUC: $66.10 \%$ )

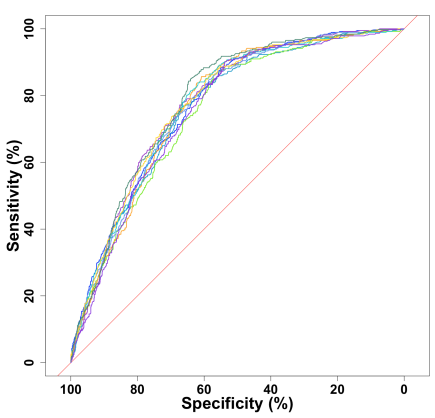

(f) $1250-1500 \mathrm{~ms}$ (AUC: $76.94 \%$ )

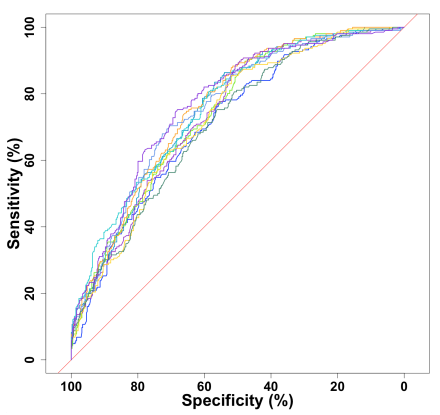

(i) $\geq 2000 \mathrm{~ms}$ (AUC: $74.14 \%$ )

Fig. 25. The Receiver Operating Characteristic (ROC) curves for the models trained on each fold and per latency bin.

The proposed technique works as follows. The click prediction model is executed at regular intervals (e.g., every 250 milliseconds) during the processing of a query. At each execution, the model provides a prediction about whether the user would issue any click on the top search results computed so far, i.e., engage with the search results. If the model predicts a click, processing of the query continues and we execute the model again at the end of the current interval. The process is repeated until the search results are fully computed or, in a particular execution of the model, we predict that the user will not issue a click on the search results. In the former case, the fully computed results are returned to the user. In the latter case, the processing of the query is 
Table 12. Possible cases

\begin{tabular}{lllll}
\hline & & & \multicolumn{2}{c}{ Measure } \\
\cline { 4 - 5 } Cases & Processing & Click & Energy consumption & User engagement \\
\hline Case I & Full & Yes & Not changed & Not degraded \\
Case II & Full & No & Not changed (missed opportunity) & Not degraded \\
Case III & Partial & No & Reduced & Not degraded \\
Case IV & Partial & Yes & Reduced & Potentially degraded \\
\hline
\end{tabular}

terminated early, and the user is presented with some partially computed results. The partial results can be improved by relying on certain cache-based query processing techniques, such as those proposed in [9]. In general, this kind of early termination can lead to energy saving because the computational resources are utilized for a shorter period of time during query processing.

Depending on whether the query is fully processed or not and our knowledge of whether the user would issue a click on the fully computed search results or not, there are four possible cases (shown in Table 12):

- Case I: The query is fully processed, and the user would issue a click on the fully computed search results. In this case, we cannot achieve any energy saving (since query processing is not terminated early), and the user experience is not degraded (since the user is served fully computed search results and issues a click).

- Case II: The query is fully processed, but the user would not issue any click on the fully computed search results. This is similar to Case I in that we cannot achieve any energy saving, and the user engagement is not degraded. However, there is a missed opportunity in reducing the energy consumption of the search engine since the processing of the query could have been terminated early with no adverse effect on user engagement.

- Case III: The processing of the query is terminated early, and the user would not issue any click if the results were fully computed. In this case, we can achieve some energy saving (since the query is not fully processed), and the user engagement is not degraded (since the user would not issue any click anyway, even on the fully computed search results).

- Case IV: The processing of the query is terminated early, but the user would issue a click if the results were fully computed. In this case, we can achieve some energy saving (since the query is not fully processed), but the user engagement may be degraded (since the user may not issue a click on the partially computed results whereas she would issue a click on the fully computed results).

\subsection{Experimental Setting}

We conduct our experiments using the query logs used in the predictive modelling task (Section 6). Following the study in [30], we assume the presence of an infinite result cache deployed in the search engine. We also assume that the time overhead and energy consumption of cache lookups are negligible and the cache hit rate is around 50\% [4]. Estimating the actual energy consumed by each query would normally involve a hardware-based study. Since this is beyond the scope of this preliminary analysis, we estimate the energy consumption using the actual query latency and considering the energy consumed by a Google search. ${ }^{8}$ We note that our evaluation focuses solely on decreasing the energy consumption of the search engine, omitting any adversarial effect on user

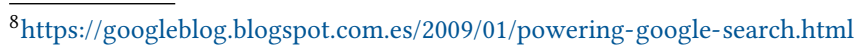


Table 13. Likelihood of different cases with respect to response latency bins

\begin{tabular}{|c|c|c|c|c|c|c|c|c|c|}
\hline \multirow[b]{2}{*}{ Cases } & \multicolumn{9}{|c|}{ Latency bin (ms) } \\
\hline & $\geq 0$ & $250-500$ & $500-750$ & $750-1000$ & $1000-1250$ & $1250-1500$ & $1500-1750$ & $1750-2000$ & $\geq 2000$ \\
\hline Case I (\%) & 42.75 & 66.18 & 33.67 & 58.57 & 52.93 & 68.06 & 66.27 & 65.11 & 54.57 \\
\hline Case II (\%) & 6.65 & 15.93 & 8.45 & 4.05 & 4.89 & 17.38 & 18.14 & 16.31 & 16.65 \\
\hline Case III (\%) & 20.95 & 13.91 & 23.18 & 16.29 & 18.42 & 7.30 & 7.62 & 9.51 & 15.29 \\
\hline Case IV (\%) & 29.65 & 3.98 & 34.69 & 21.09 & 23.76 & 7.27 & 7.97 & 9.07 & 13.49 \\
\hline
\end{tabular}

Table 14. Energy saving per day for queries in different response latency bins

\begin{tabular}{lrrrrrrrrr}
\hline Energy & \multicolumn{10}{c}{ Latency bin $(\mathrm{ms})$} \\
\cline { 2 - 10 } consumption & $\geq 0$ & $250-500$ & $500-750$ & $750-1000$ & $1000-1250$ & $1250-1500$ & $1500-1750$ & $1750-2000$ & $\geq 2000$ \\
\hline Original (MWh) & 235.54 & 140.18 & 197.14 & 286.04 & 388.19 & 464.73 & 503.84 & 581.86 & 883.90 \\
Reduced (MWh) & 124.70 & 117.49 & 84.68 & 183.27 & 229.44 & 401.33 & 427.35 & 477.01 & 683.88 \\
Saving (\%) & 47.06 & 16.18 & 57.04 & 35.93 & 40.90 & 13.64 & 15.18 & 18.02 & 22.63 \\
\hline
\end{tabular}

experience (due to potential degradation in search result quality). Evaluation of the latter aspect is left as future work due to current unavailability of click data for partially computed search results.

\subsection{Experimental Results}

Using the experimental setting described in the previous section, we simulate a baseline approach where the queries are processed without any early termination and measure the total energy expenditure for processing all queries. In the same way, we compute the energy expenditure by the proposed query processing approach, which employs early termination (Section 7.1). We perform the simulation across the nine different response latency bins shown in Table 13. We estimate the daily energy consumption (in MWh) and the reduction that can be achieved (in MWh), based on the query traffic processed by Google. ${ }^{9}$

Table 13 shows the percentage of queries falling under a particular case. The cases displayed in this table can provide us useful bounds. For example, although we do not have a ground truth for the clicks on partial results, we can get an upper bound for the potential degradation in user engagement by considering the number of queries under Case IV. The degradation in user engagement can be mitigated by devising mechanisms for accurate prediction of user-perceived response latency, such as, for example, training models on a per-user basis that consider demographic (age, gender) or contextual (time, location, weather) factors.

Table 14 shows the results of our simulation. According to this table, the percentage of energy saving ranges between $13.64 \%$ and $57.04 \%$, depending on the latency bin we consider. The largest saving is achieved for queries that fall in the 500-1250 ms range. However, the remaining bins also present substantial potential for energy savings. This preliminary analysis highlights the benefits of using search response latency in click prediction and demonstrates the potential for achieving energy savings in commercial web search engines.

\section{CONCLUSIONS}

This paper is the outcome of an attempt to understand the response latency issue in web search engines. As our first contribution, we conducted some experiments, using a large, real-life query $\log$ data, to characterise the response latency of a commercial web search engine. We then focused on the impact of response latency on user behaviour. To this end, we conducted a controlled user

\footnotetext{
${ }^{9}$ https://www.comscore.com/Insights/Rankings/comScore-Releases-February-2016-US-Desktop-Search-Engine-Rankings
} 
study and also performed a large-scale query log analysis. The user study revealed that up to a certain point $(500 \mathrm{~ms})$ the added response time delays are not noticeable by the users. However, after a certain point $(1000 \mathrm{~ms})$, the users could feel the added delays with very high likelihood. The query log analysis also revealed interesting findings about the change in user behaviour as latency increases. In particular, given two content-wise identical search result pages, we showed that the users are more likely to perform clicks on the result page that is served with lower latency. Moreover, additional analyses indicated that the degree to which response latency affects the click behaviour varies with the user, query, and context attributes. Finally, we presented a machine learning framework that leverages the latency information in a click prediction task. Through carefully conducted experiments, we demonstrated the predictive power of this model and applied it to a use case about achieving energy savings in web search engines.

We believe that the subjective nature of perceived latency creates an opportunity for search engines. Search results can be served to each user at custom latencies depending on the estimated behavioural impact on the user. For example, if no negative impact is estimated on the user experience, search results may be served with high latencies by computing them using less resources. Serving results at right latencies may bring further financial benefits to search engines in the form of decreased hardware investments and reduced energy consumption. The machine learning framework we devised in this work and alike can be useful in such tasks. As a separate research problem, the existing prediction models for query processing latency can be extended to predict the end-to-end, user-perceived latency values. Such models can find better use in practical search systems.

\section{REFERENCES}

[1] Ioannis Arapakis, Xiao Bai, and B. Barla Cambazoglu. 2014. Impact of Response Latency on User Behavior in Web Search. In Proceedings of the 37th International ACM SIGIR Conference on Research and Development in Information Retrieval (SIGIR '14). 103-112.

[2] Leif Azzopardi. 2011. The economics in interactive information retrieval. In Proc. 34th Int'l ACM SIGIR Conf. Research and Development in Information Retrieval. 15-24.

[3] Leif Azzopardi, Diane Kelly, and Kathy Brennan. 2013. How query cost affects search behavior. In Proc. 36th Int'l ACM SIGIR Conf. Research and Development in Information Retrieval. 23-32.

[4] Ricardo Baeza-Yates, Aristides Gionis, Flavio Junqueira, Vanessa Murdock, Vassilis Plachouras, and Fabrizio Silvestri. 2007. The Impact of Caching on Search Engines. In Proceedings of the 30th Annual International ACM SIGIR Conference on Research and Development in Information Retrieval (SIGIR '07). ACM, New York, NY, USA, 183-190.

[5] Miguel Barreda-Ángeles, Ioannis Arapakis, Xiao Bai, B. Barla Cambazoglu, and Alexandre Pereda-Baños. 2015. Unconscious physiological effects of search latency on users and their click behaviour. In Proc. 38th Int'l ACM SIGIR Conf. Research and Development in Information Retrieval. 203-212.

[6] Feza Baskaya, Heikki Keskustalo, and Kalervo Järvelin. 2012. Time drives interaction: Simulating sessions in diverse searching environments. In Proc. 35th Int'l ACM SIGIR Conf. Research and Development in Information Retrieval. 105-114.

[7] Y. Benjamini and Y. Hochberg. 1995. Controlling the false discovery rate - a practical and powerful approach to multiple testing. Journal of the Royal Statistical Society, Series B 57, 1 (1995), 289-300.

[8] Jake D. Brutlag, Hilary Hutchinson, and Maria Stone. 2008. User preference and search engine latency. In Proceedings of the ASA foint Statistical Meetings.

[9] B. Barla Cambazoglu, Ismail Sengor Altingovde, Rifat Ozcan, and Ȯzgür Ulusoy. 2012. Cache-Based Query Processing for Search Engines. ACM Trans. Web 6, 4, Article 14 (Nov. 2012), 24 pages.

[10] B. Barla Cambazoglu and Ricardo A. Baeza-Yates. 2015. Scalability Challenges in Web Search Engines. Morgan \& Claypool Publishers.

[11] Matteo Catena, Craig Macdonald, and Nicola Tonellotto. 2015. Load-sensitive CPU Power Management for Web Search Engines. In Proceedings of the 38th International ACM SIGIR Conference on Research and Development in Information Retrieval (SIGIR '15). ACM, New York, NY, USA, 751-754.

[12] N. V. Chawla, K. W. Bowyer, L. O. Hall, and W. P. Kegelmeyer. 2002. SMOTE: Synthetic Minority Over-Sampling Technique. Journal of Artificial Intelligence Research 16, 321-357 (2002). 
[13] Haibin Cheng and Erick Cantú-Paz. 2010. Personalized Click Prediction in Sponsored Search. In Proceedings of the Third ACM International Conference on Web Search and Data Mining (WSDM '10). ACM, New York, NY, USA, 351-360.

[14] Gobinda Chowdhury. 2012. An agenda for green information retrieval research. Inf. Process. Manage. 48, 6 (2012), 1067-1077.

[15] Jim Dabrowski and Ethan V. Munson. 2011. 40 years of searching for the best computer system response rime. Interact. Comput. 23, 5 (2011), 555-564.

[16] Erica S. Davis and Donald A. Hantula. 2001. The effects of download delay on performance and end-user satisfaction in an Internet tutorial. Computers in Human Behavior 17, 3 (2001), 249-268.

[17] B. G. C. Dellaert and B. E. Kahn. 1999. How tolerable is delay: Consumers' evaluations of Internet web sites after waiting. Journal of Interactive Marketing 13, 1 (1999), 41-54.

[18] Alan R. Dennis and Nolan J. Taylor. 2006. Information foraging on the Web: The effects of "acceptable" Internet delays on multi-page information search behavior. Decision Support Systems 42, 2 (2006), 810-824.

[19] Ana Freire, Craig Macdonald, Nicola Tonellotto, Iadh Ounis, and Fidel Cacheda. 2014. A Self-adapting Latency/Power Tradeoff Model for Replicated Search Engines. In Proceedings of the 7th ACM International Conference on Web Search and Data Mining (WSDM '14). ACM, New York, NY, USA, 13-22.

[20] Ana Freire, Craig Macdonald, Nicola Tonellotto, Iadh Ounis, and Fidel Cacheda. 2015. Queuing Theory-based Latency/Power Tradeoff Models for Replicated Search Engines. 21, 13 (dec 2015), 1790-1809.

[21] Dennis F. Galletta, Raymond Henry, Scott McCoy, and Peter Polak. 2003. Web site delays: How tolerant are users? Journal of the Association for Information Systems 5 (2003), 1-28.

[22] J. Gwizdka and I. Lopatovska. 2009. The role of subjective factors in the information search process. fournal of the American Society for Information Science and Technology 60, 12 (2009), 2452-2464.

[23] Marc Hassenzahl. 2004. Funology. In The Thing and I: Understanding the Relationship Between User and Product, Mark A. Blythe, Kees Overbeeke, Andrew F. Monk, and Peter C. Wright (Eds.). Kluwer Academic Publishers, 31-42.

[24] Xinran He, Junfeng Pan, Ou Jin, Tianbing Xu, Bo Liu, Tao Xu, Yanxin Shi, Antoine Atallah, Ralf Herbrich, Stuart Bowers, and Joaquin Quiñonero Candela. 2014. Practical Lessons from Predicting Clicks on Ads at Facebook. In Proceedings of the Eighth International Workshop on Data Mining for Online Advertising (ADKDD'14). ACM, New York, NY, USA, Article 5, 9 pages.

[25] Seung-Won Hwang, Saehoon Kim, Yuxiong He, Sameh Elnikety, and Seungjin Choi. 2016. Prediction and Predictability for Search Query Acceleration. ACM Transactions on the Web (TWEB) 10, 3 (2016), 19.

[26] Julie A. Jacko, Andrew Sears, and Michael S. Borella. 2000. The effect of network delay and media on user perceptions of web resources. Behaviour \& Information Technology 19, 6 (2000), 427-439.

[27] Vidit Jain and Manik Varma. 2011. Learning to Re-rank: Query-dependent Image Re-ranking Using Click Data. In Proceedings of the 20th International Conference on World Wide Web (WWW'11). ACM, New York, NY, USA, 277-286.

[28] Kalervo Järvelin and Jaana Kekäläinen. 2002. Cumulated gain-based evaluation of IR techniques. ACM Trans. Inf. Syst. 20, 4 (2002), 422-446.

[29] Myeongjae Jeon, Saehoon Kim, Seung-won Hwang, Yuxiong He, Sameh Elnikety, Alan L. Cox, and Scott Rixner. 2014. Predictive parallelization: taming tail latencies in web search. In Proceedings of the 37th International ACM SIGIR Conference on Research \& Development in Information Retrieval. 253-262.

[30] Enver Kayaaslan, B. Barla Cambazoglu, Roi Blanco, Flavio P. Junqueira, and Cevdet Aykanat. 2011. Energy-price-driven Query Processing in Multi-center Web Search Engines. In Proceedings of the 34th International ACM SIGIR Conference on Research and Development in Information Retrieval (SIGIR '11). ACM, New York, NY, USA, 983-992.

[31] Saehoon Kim, Yuxiong He, Seung-won Hwang, Sameh Elnikety, and Seungjin Choi. 2015. Delayed-dynamic-selective (DDS) prediction for reducing extreme tail latency in web search. In Proceedings of the Eighth ACM International Conference on Web Search and Data Mining. 7-16.

[32] James R. Lewis. 1995. IBM Computer Usability Satisfaction Questionnaires: Psychometric Evaluation and Instructions for Use. International fournal of Human-Computer Interaction 7, 1 (1995), 57-78.

[33] Irene Lopatovska. 2009. Searching for good mood: examining relationships between search task and mood. Proceedings of the American Society for Information Science and Technology 46, 1 (2009), 1-13.

[34] Craig Macdonald, Nicola Tonellotto, and Iadh Ounis. 2012. Learning to predict response times for online query scheduling. In Proceedings of the 35th International ACM SIGIR Conference on Research and Development in Information Retrieval. 621-630.

[35] David Maxwell and Leif Azzopardi. 2014. Stuck in traffic: How temporal delays affect search behaviour. In Proceedings of the 5th Information Interaction in Context Symposium (IIiX '14). ACM, New York, NY, USA, 155-164.

[36] Lori McCay-Peet, Mounia Lalmas, and Vidhya Navalpakkam. 2012. On Saliency, Affect and Focused Attention. In ACM SIGCHI Conference on Human Factors in Computing Systems (CHI '12). ACM, New York, NY, USA, 541-550. 
[37] H. Brendan McMahan, Gary Holt, D. Sculley, Michael Young, Dietmar Ebner, Julian Grady, Lan Nie, Todd Phillips, Eugene Davydov, Daniel Golovin, Sharat Chikkerur, Dan Liu, Martin Wattenberg, Arnar Mar Hrafnkelsson, Tom Boulos, and Jeremy Kubica. 2013. Ad Click Prediction: A View from the Trenches. In Proceedings of the 19th ACM SIGKDD International Conference on Knowledge Discovery and Data Mining (KDD '13). ACM, New York, NY, USA, 1222-1230.

[38] Alistair Moffat and Justin Zobel. 2008. Rank-biased precision for measurement of retrieval effectiveness. ACM Trans. Inf. Syst. 27, 1 (2008), 2:1-2:27.

[39] Fiona Fui-Hoon Nah. 2004. A study on tolerable waiting time: how long are Web users willing to wait? Behaviour and Information Technology 23, 3 (2004), 153-163.

[40] Heather L. O'Brien and Elaine G. Toms. 2010. The development and evaluation of a survey to measure user engagement. fournal of the American Society for Information Science and Technology 61, 1 (January 2010), 50-69.

[41] Judith Ramsay, Alessandro Barbesi, and Jenny Preece. 1998. A psychological investigation of long retrieval times on the World Wide Web. Interacting with Computers 10, 1 (1998), 77-86. arXiv:http://iwc.oxfordjournals.org/content/10/1/77.full.pdf+html

[42] E. Schurman and J. Brutlag. 2009. Performance related changes and their user impact. In Velocity - Web Performance and Operations Conf.

[43] M. D. Smucker. 2009. Towards timed predictions of human performance for interactive information retrieval evaluation. In Proc. Symp. Human-Computer Information Retrieval.

[44] Mark D. Smucker and Charles L.A. Clarke. 2012. Time-based calibration of effectiveness measures. In Proc. 35th Int'l ACM SIGIR Conf. Research and Development in Information Retrieval. 95-104.

[45] Nolan J. Taylor, Alan R. Dennis, and Jeff W. Cummings. 2013. Situation normality and the shape of search: The effects of time delays and information presentation on search behavior. Journal of the American Society for Information Science and Technology 64, 5 (2013), 909-928.

[46] Jaime Teevan, Kevyn Collins-Thompson, Ryen W. White, Susan T. Dumais, and Yubin Kim. 2013. Slow search: Information retrieval without time constraints. In Proceedings of the Symposium on Human-Computer Interaction and Information Retrieval (HCIR '13). ACM, New York, NY, USA, Article 1, 10 pages.

[47] Edmund R. Thompson. 2007. Development and Validation of an Internationally Reliable Short-Form of the Positive and Negative Affect Schedule (PANAS). Fournal of Cross-Cultural Psychology 38, 2 (2007), 227-242.

Received November 2016; revised December 2016; revised May 2017; accepted June 2017 